\title{
On the Sensitivity of Deep-Convection Initiation to Horizontal Grid Resolution
}

\author{
Siu Lung Tang \\ Department of Atmospheric and Oceanic Sciences, \\ McGill University, Montreal
}

A thesis submitted to McGill University in partial fulfillment of the requirements of the degree of Master of Science

July 2019

(C) Siu Lung Tang 2019 


\section{Acknowledgements}

I would like to express my gratitude and appreciation to Prof. Daniel Kirshbaum for his guidance over the course of this thesis. He has provided me with an invaluable opportunity to pursue scientific research on moist convection initiation. This work would not have been completed without his feedback and encouragement.

I would like to extend my gratitude to George Bryan for developing the CM1 model (release 19.4) and making it available to everyone.

I have benefited from many useful discussions with my friends at the AOS department, in particular, David, Yuwei, and Sonja for providing insights that expedited the progress of my project. I want to thank Pascal for providing assistance in numerical simulations and coursework, and translating the Abstract into French.

I am also grateful to my family and friends for their continued support. 


\section{Abstract}

Numerically simulated moist convection exhibits strong dependence on model grid resolution. With the use of a cloud-resolving model, the effect of horizontal grid spacing $(\Delta)$ on deep convection initiation is investigated to identify the cloud-layer and subcloud processes regulating this sensitivity. Simulations are initialized with sounding representative of a quiescent conditionally unstable flow. A prescribed diurnal surface heating function is adopted to generate a long surface-based convergence line, with an associated updraft that breaches the LFC to initiate cumulus convection.

Results from simulations with $\Delta$ systemically varied between $125 \mathrm{~m}$ and $500 \mathrm{~m}$ reveal that cloud-top height in the $250-\mathrm{m}$ and $500-\mathrm{m}$ cases $(8-9 \mathrm{~km})$ are substantially greater than that in the $125-\mathrm{m}$ case $(6-7 \mathrm{~km})$. In the cloud layer, the differences in conditionally averaged core properties (vertical velocity $(w)$, buoyancy $(b)$, and hydrometeor content $\left.\left(q_{h}\right)\right)$ within the cloud cores are rather limited. However, significant variations in cloudcore vertical mass flux is found, with the 250-m run having the strongest mass flux near cloud base and the 125-m run having the weakest at all heights, suggesting that the intensity of subcloud updraft penetrating into the cloud layer from below regulates the core mass flux within the cloud layer, and hence the cloud-top height. Parcel trajectories indicate that subcloud parcels increasingly struggle to reach the lifted condensation level (LCL) as $\Delta$ is varied from $250 \mathrm{~m}$. Part of this finding stems from enhanced $(\sim 33 \%$ more) adverse vertical pressure perturbation gradient exerting on the lower half of the buoyant updrafts in the 125-m and 500-m cases, which display weaker and wider subcloud updrafts than the 250-m case. Analysis of the Reynolds-averaged momentum equations demonstrates that total (subgrid plus resolved) turbulent forcings on both vertical $(w)$ and zonal $(u)$ velocities are enhanced in the 125 -m case, which reduces vertical motion within the updraft core but increases $w$ on the updraft flanks. Such distortion of updraft structure in the 500-m case, contrarily, is due to its limitations in resolving the narrow (width $\sim 1 \mathrm{~km}$ ) updraft adequately on the model grids. Thus, relative to the $250-\mathrm{m}$ run, the more hydrostatic and diffuse updrafts in the $125-\mathrm{m}$ and 500-m runs acquire less vertical acceleration, which, in turn, weakens the core-base mass flux. Nonetheless, decrease in core mass flux with height is the slowest in the 500-m run, in that midlevel detrainment associated with the case is the weakest among them all. Consequently, the 
cloud-top height is similar in the $250-\mathrm{m}$ and $500-\mathrm{m}$ cases but radically lower in the $125-\mathrm{m}$ case. 


\section{Résumé}

La convection humide simulée numériquement présente une forte dépendance à la résolution de la grille du modèle. Avec l'utilisation d'un modèle résolvant les nuages, l'effet de l'espacement de grille horizontal $(\Delta)$ sur l'initiation de la convection profonde est étudié afin d'identifier les processus des couches nuageuse et sous-nuage régulant cette sensibilité. Les simulations sont initialisées avec un profil représentant un écoulement conditionnellement instable au repos. Une fonction de chauffage de surface diurne est prescrite pour générer une longue ligne de convergence à la surface, avec un courant ascendant associé qui dépasse le LFC pour initier la convection de cumulus.

Les résultats de simulations avec $\Delta$ variant systématiquement entre $125 \mathrm{~m}$ et $500 \mathrm{~m}$ révèlent que la hauteur du sommet des nuages dans les tranches de 250 et 500 m $(8-9$ km) est nettement supérieure à celle dans le cas à $125 \mathrm{~m}(6-7 \mathrm{~km})$. Dans la couche nuageuse, les différences dans les propriétés centrales conditionnellement moyennées (vitesse verticale $(w)$, flottabilité $(b)$ et contenu d'hydrométéore $\left.\left(q_{h}\right)\right)$ au sein des noyaux de nuage sont plutôt limitées. Cependant, on observe des variations significatives du flux de masse vertical des noyaux de nuages: le cas à $250 \mathrm{~m}$ présentant le plus fort flux de masse près de la base du nuage et celui à $125 \mathrm{~m}$ le plus faible à toutes les hauteurs, ce qui suggère que l'intensité du courant ascendant sous-nuage qui pénétre dans la couche nuageuse par en bas régule le flux de masse du noyau dans la couche nuageuse et, par conséquent, la hauteur du sommet du nuage. La trajectoire des parcelles indique que les parcelles de sous-nuage peinent de plus en plus à atteindre le niveau de condensation par ascension (LCL) lorsque $\Delta$ s'éloigne de $250 \mathrm{~m}$. Une partie de cette constatation découle d'un gradient de perturbation de pression vertical défavorable renforcé ( 33\% plus grand) s'exerçant sur la moitié inférieure des courants ascendants flottants dans les cas à 125 et $500 \mathrm{~m}$, qui présentent des courants ascendants sous-nuage plus faible et plus large que le cas à $250 \mathrm{~m}$. L'analyse des équations de la quantité de mouvement sous la moyenne de Reynolds montre que les forçages turbulents totaux (résolus et paramètrisés) sur les vitesses verticale $(w)$ et zonale $(u)$ sont augmentés dans le cas à $125 \mathrm{~m}$, ce qui réduit les mouvements verticaux dans le coeur du courant ascendant mais augmente $w$ sur ses flancs. Une telle distorsion de la structure du courant ascendant dans le cas à $500 \mathrm{~m}$ est, au contraire, due à son incapacité à résoudre correctement le courant ascendant 
étroit (largeur $\sim 1 \mathrm{~km}$ ) sur les grilles du modèle. Par conséquent, par rapport au cas à $250 \mathrm{~m}$, les courants ascendants plus hydrostatiques et diffus dans les cas à 125 et $500 \mathrm{~m}$ acquièrent une accélération verticale moindre, ce qui affaiblit le flux de masse noyau-base. Néanmoins, la diminution du flux de masse du noyau avec la hauteur est la plus lente dans le cas à $500 \mathrm{~m}$, dans la mesure où le détraînment à mi-hauteur de ce cas est le plus faible de tous. Par conséquent, la hauteur du sommet des nuages est similaire pour les cas à $250 \mathrm{~m}$ et à $500 \mathrm{~m}$ mais radicalement inférieure pour le cas à $125 \mathrm{~m}$. 


\section{Contents}

Acknowledgements $\quad$ i

Abstract $\quad$ ii

Résumé $\quad$ iv

1 Introduction $\quad 1$

2 Numerical Simulations $\quad 9$

2.1 Model setup . . . . . . . . . . . . . . . . . . . . . . . . . 9 9

2.2 Overview of results . . . . . . . . . . . . . . . . . . . . 12

3 Physical Interpretation of Results 16

3.1 Cloud-layer analysis . . . . . . . . . . . . . . . . 16

3.2 Parcel trajectories . . . . . . . . . . . . . . . . 20

3.3 Subcloud-layer analysis . . . . . . . . . . . . . . . . . . . . 22

3.3.1 The $w$ budget . . . . . . . . . . . . . . . . . . . 23

3.3 .2 Updraft morphology . . . . . . . . . . . . . . . 28

3.3 .3 The role of turbulence . . . . . . . . . . . . . . . . . . . 31

3.3.3.1 Vertical velocity . . . . . . . . . . . . 32

3.3.3.2 Horizontal velocity . . . . . . . . . . . . . . 37

4 Conclusions $\quad 39$

$\begin{array}{ll}\text { References } & 43\end{array}$ 


\section{List of Figures}

1 Adapted from Fig. 10 of Bryan et al. (2003), inertial subrange of turbulent eddies are only captured in the $125-\mathrm{m}$ and $250-\mathrm{m}$ simulations. . . . . . .

2 Adapted from Figs. 10 and 16 of Rousseau-Rizzi et al. (2017), scatterplots of final cloud height and initial core at $\Delta=$ (left) $125 \mathrm{~m}$, (middle) $250 \mathrm{~m}$, and (right) $500 \mathrm{~m}$.

3 (top) Contours of surface heating function $\left(\mathrm{Wm}^{-2}\right)$ on $x-y$ plane. (bottom) Distribution of Gaussian-shaped surface heat flux across the $x$-dimension. . . . . . . . . . . . . . . . . . .

4 Adapted from Fig. 1b of Rousseau-Rizzi et al. (2017), skew T-log $p$ initial sounding of background temperature (thick blue), background dewpoint (dashed blue), and ascent temperature (thick black).

$5 \quad$ Isosurface (grey contours) of $q_{c}+q_{r}$ equal to $0.1 \mathrm{gkg}^{-1}$ at (a) 04:00, (b) 05:00, and (c) 06:00 for $\Delta=125 \mathrm{~m}$. The same quantities for $\Delta=250$ $m$ are shown in panels (d-f), and for $\Delta=500 m$ are shown in panels (g-i). Red contours denote perturbation of potential temperature with respect to mean in $x$-direction on the surface. Black arrows denote surface horizontal wind. . . . . . . . . . . . . . . . . . . .

6 Time series of (a) magnitude of surface convergence, averaged in $y$ along the convergence line and in $x$ over the central $1 \mathrm{~km}$ of the domain; (b) pseudoadiabatic mean-layer (500-m) CAPE and CIN over the central $1 \mathrm{~km}$ of the convergence line; (c) maximum cloud-top height at which $q_{h}=q_{c}+q_{r}+q_{i}+q_{s}+q_{g}$ exceeds $0.1 \mathrm{gkg}^{-1}$ with LNB overlaid in black; and (d) midlevel relative humidity, RH, calculated by volume-weighted average over the central $5 \mathrm{~km}$ and between heights of 3 and $8 \mathrm{~km}$ of the domain.

7 Profiles of (a) total core mass flux, (b) total area, (c) average vertical velocity $\bar{w},(\mathrm{~d})$ average buoyancy $\bar{b}$, and (e) average hydrometero mixing ratio $\overline{q_{h}}$, of cores over the whole domain from 05:00 to 06:00. LCL is indicated by dashed black lines. . . . . . . . . . . . . . . . 
For control case, profile of (a) fractional entrainment rate $\bar{\epsilon}$ and (b) fractional detrainment rate $\bar{\delta}$. The quantities are averaged over number of convective cores from 05:00 to 06:00. Results obtained from simulation with horizontal grid spacing $\Delta$ equal to $125 \mathrm{~m}$ are shown in dash-dot green lines, those obtained with $\Delta=250 \mathrm{~m}$ are shown in solid blue lines, and those with $\Delta$ equal to $500 \mathrm{~m}$ are shown in dashed red lines. . . . . .

9 (a) Distribution of vertical velocity $w_{\mathrm{LCL}}$ of parcels prior to crossing the LCL for the last time. The number of parcels in each velocity bin is $\mathrm{N}_{\mathrm{w}}$ and the total number of parcels reaching the LCL in each simulation is $\mathrm{N}_{\mathrm{LCL}}$. (b) Percentage (\%) of parcels having maximum height greater than or equal to the value denoted by each bin on the $y$-axis, among the subset of parcels that ever entered the CCR and reached the LCL . . . . . . . .

10 Contours of $y$-averaged $w$ (filled with greyscale contours) and $y$-averaged wind vectors (blue arrows) over the time interval stated from simulation with grid-spacing of (a) $\Delta=125 \mathrm{~m}$, (b) $\Delta=250 \mathrm{~m}$, and (c) $\Delta=500 \mathrm{~m}$. LCL is indicated by dashed black lines. Contour lines range from $0 \mathrm{~ms}^{-1}$ to $4 \mathrm{~ms}^{-1}$ with $0.5 \mathrm{~ms}^{-1}$ increments. . . . . . . . . . . . .

11 Profile of each term in $w$-budget model output averaged horizontally over the CCR and temporally from 05:00 to 06:00.

12 Comparison of (a) the RHS forcing terms of (6) to (b) the LHS timetendency of (6) between the three cases. The profiles are averaged horizontally over the CCR and temporally over 05:00 to 06:00. . . . . . .

13 Contours of $y$ - and time-averaged (05:00-06:00) buoyancy term in (6). Panel (b) shows contours of buoyancy term when $\Delta=250 \mathrm{~m}$. Panels (a) and (c) show differences in buoyancy between $\Delta=125 \mathrm{~m}$ and $\Delta=250 \mathrm{~m}$ cases and $\Delta=500 m$ and $\Delta=250 m$ cases respectively. . . . . . . 26

14 As in Fig. 13, but for VPPG term in (6) . . . . . . . . . . . . . 27

15 Profiles of total TKE $\left(\mathrm{TKE}_{\mathrm{tot}}=\mathrm{TKE}_{\mathrm{sgs}}+\mathrm{TKE}_{\mathrm{res}}\right)$, averaged temporally from 05:00 to 06:00 and spatially over the CCR, for the three highestresolution runs. . . . . . . . . . . . . . . . . . 
16 Distributions of $y$-averaged resolved turbulent fluxes of (a), (c), (e) $u^{\prime} w^{\prime}$ and (b), (d), (f) $w^{\prime} w^{\prime}$. The absolute fluxes at $\Delta=250 \mathrm{~m}$ are shown at (c) and (d). The difference of resolved fluxes between $\Delta=125 \mathrm{~m}$ and $\Delta=250 \mathrm{~m}$ are shown in (a-b) and between $\Delta=500 \mathrm{~m}$ and $\Delta=250 \mathrm{~m}$ are shown in $(\mathrm{e}-\mathrm{f}) \ldots \ldots \ldots \ldots \ldots \ldots$

17 Total (subgrid plus resolved) turbulent forcing on $w$ for (a) $\Delta=125 \mathrm{~m}-$ $\Delta=250 m$, (b) $\Delta=250 m$, and (c) $\Delta=500 m-\Delta=250 m \ldots 35$

18 As in Fig. 17, but for the (a), (c), (e) horizontal and (b), (d), (f) vertical components of the total turbulent forcing on $w \ldots \ldots 36$

19 As in Fig. 17, but for turbulent foricng on $u$. . . . . . . . . . . . 38

\section{List of Tables}

1 Statistics of parcel-trajectory analyses in the 125-m, 250-m, and 500-m runs. In the left column, $\mathrm{N}$ is the total number of parcels entering the central converging region (CCR), $\mathrm{N}_{\mathrm{LCL}}$ is the number of parcels entering the CCR that reach the LCL, and $\mathrm{N}_{\text {core }}$ is the number of parcels that cross the LFC to become a convective core. . . . . . . . . . . . . 20

2 Comparison of subcloud updraft width $(L)$ and depth $(H)$, and squared aspect ratio $\left((H / L)^{2}\right)$ between four simulations with different horizontal grid spacings. . . . . . . . . . . . . . . . . 


\section{Introduction}

Moist convection is fundamental in regulating a variety of atmospheric phenomena ranging from global-scale circulations to precipitating cumulus clouds with sub-kilometre horizontal length scales. On the global scale, insolation in the tropics creates low pressure, causing easterly winds from both hemispheres to converge and force conditionally unstable airmasses in place to ascend to the tropopause. This constitutes the ascending branch of the Hadley cell, which is one of the prominent large scale flows responsible for transporting heat from low to high latitudes. It is also crucial for governing weather patterns over the tropical and subtropical zones, home to the majority of the world's population. The rising motion brings cloudy and rainy weather to the affected regions where significant precipitation and thunderstorms happen frequently throughout the year.

The contribution of moist convection to severe weather is also critically important. Under certain environmental conditions, cumuli may vertically develop into cumulonimbus clouds and thunderstorms, which are capable of generating heavy rainfall and lightning. With sufficient vertical wind shear, the storms could evolve into supercell thunderstorms. The associated intense, rapidly rotating updrafts can produce hail, downburst, and tornadoes which threatens life and property.

Despite a large amount of research devoted to improving our knowledge of the mechanism governing convective development, predicting accurately the location and timing of initiation of convection remains a challenging problem (e.g., Weckwerth and Parsons 2006; Kang and Bryan 2011). Part of the difficulty stems from our lack of understanding of parameters controlling subgrid-scale and microphysical processes involved in convection, which impedes realistic representation of the processes through parameterizations used in weather prediction models. Bechtold et al. (2004) studied the diurnal cycle of convection over the Amazon basin and tropical Africa. Their general circulation model showed difficulty in resolving the rapidly developing convection that was driven locally by solar heating. This led to the time of maximum rainfall predicted numerically to be several hours earlier than that shown by observations. Even with the advancement of convective parameterization schemes over the years, model biases and errors in simulating the diurnal cycle persist, with a tendency of having the onset of precipitation and the development of deep convection out of phase with observations (Stratton and Stirling 
2012; Bechtold et al. 2014).

The absence of high-resolution measurements of kinematic and thermodynamic variables, particularly the distribution of water vapor on fine temporal and spatial scales, in the lower troposphere is considered one of the major factors in limiting the accuracy of predicting convective precipitation (e.g. Weckwerth and Parsons 2006; Bennett et al. 2006; Fabry 2006). The significance of measurement uncertainty is highlighted in a numerical study of moist convection conducted by Crook (1996). He showed that initiation of convection is highly sensitive to the spatial variations of temperature and moisture fields in the boundary layer. Specifically, he found that perturbations of $1{ }^{\circ} \mathrm{C}$ in surface temperature and $1 \mathrm{gkg}^{-1}$ in surface moisture determine the difference between well-developed convection and no convection at all.

Initiation of moist convection requires the presence of three ingredients: a conditionally unstable atmosphere, adequate supply of low-level moisture, and a lifting mechanism. Conditionally unstable air is characterized by an environmental lapse rate that falls inbetween the dry and moist adiabatic lapse rates. When initially unsaturated parcels are lifted through a conditionally unstable atmosphere, they acquire negative buoyancy prior to saturation. Thus, additional lifting within the layer is required to eliminate the negative buoyancy. The height at which the lifted parcel reaches saturation is called the lifting condensation level (LCL), and the height at which it becomes positively buoyant is called the level of free convection (LFC). Provision of moisture near the surface is necessary for moist convection because large upward displacements are required to bring the air to saturation. Parcels with high humidity are more readily to reach saturation, they have lower LCL and higher likelihood of becoming positively buoyant than their drier counterparts.

Two important metrics are used when determining the likelihood of convection initiation. The integrated negative buoyancy of an air parcel between the surface and the LFC is defined as the convective inhibition (CIN), which is the amount of work required to forcibly overcome the negative buoyancy. The integrated positive buoyancy of an air parcel between its LFC and the level of neutral buoyancy (LNB) is the convective available potential energy (CAPE), which is a measure of the amount of kinetic energy that an adiabatic parcel can gain from its conditionally unstable environment. Atmospheric profiles with high CAPE (usually on the order of $1000 \mathrm{~J} \mathrm{~kg}^{-1}$ or more) and low CIN 
(around $20 \mathrm{~J} \mathrm{~kg}^{-1}$ or less) provide favourable conditions for deep parcel ascent. However, they do not guarantee that the convection will actually initiate, because non-adiabatic processes can stifle vertical development.

Maintenance of positive buoyancy over a deep layer is crucial to initiate and sustain deep convection. One of the main threats to parcel ascent is entrainment of dry environmental air, which depletes buoyancy of parcels by dilution and evaporative cooling. Some numerical studies of moist convection have highlighted the role of cloud horizontal area in regulating entrainment. In general, larger clouds are less susceptible to entrainment and thus more capable of retaining higher buoyancy and vertical velocities (e.g., Kuang and Bretherton 2006; Khairoutdinov and Randall 2006). In particular, when investigating the relative importance between cloud-base and cloud-layer properties on shallow cumulus in Large Eddy Simulations (LES), Dawe and Austin (2012) showed that interior cloud parcels are more likely to have higher total water mixing ratio $\left(q_{t}\right)$ than parcels on the periphery of the cloud. This suggests that larger clouds are more effective at sheltering interior parcels from mixing with the environment and therefore are more likely to attain greater maximum heights. In simulations studying the impact of mesoscale ascent on the intensity of trade wind cumuli, Kirshbaum and Grant (2012) arrived at a similar conclusion by showing that profiles of maximum buoyancy, vertical velocity perturbation, and liquid water mixing ratio within buoyant cloud cores become increasingly moist-adiabatic as core size increases. Thus, high entrainment rates associated with narrower clouds limits their vertical motion and may inhibit the transition from shallow to deep convection (Khairoutdinov and Randall 2006; Kuang and Bretherton 2006).

The effect of entrainment can be partially mitigated by increasing moisture content in the midtrposphere, because entraining air with high relative humidity limits the degree of cloud dilution. Waite and Khouider (2010) studied the effect of environmental moisture on cloud depth. They identified that deepening of clouds depends primarily on midlevel moistening by detraining water vapor from congestus clouds. Similarly, in idealized simulations of deep convection developed in thermally driven mountain flow, Kirshbaum (2011) found that transition to deep convection requires a succession of updrafts rising through areas saturated by decaying cumuli, which diminishes the suppressive effects of evaporative cooling.

Surface-based horizontal mass convergence is associated with low-level vertical mo- 
tion, according to the continuity equation for an incompressible fluid. Above such convergent regions, the boundary-layer depth and moisture content are locally enhanced (e.g., Markowski and Richardson 2010). Rising parcels above such convergence lines thus realize less dilution and less inhibition as they ascend to the LFC. Hence, low-level convergence acts to intensify convection by strengthening inflow into the clouds from below as well as preconditioning the local environment. In an analysis of radar data over Colorado, Wilson and Schreiber (1986) found that around $80 \%$ of storms initiated were found to occur in close proximity to radar-observed boundary layer convergence lines.

Various studies have shown that regions of low-level convergence act as precursors to deep convection in conditionally unstable environments (e.g., Weckwerth and Parsons 2006; Waite and Khouider 2010; Kang and Bryan 2011). Using observations and LES simulations of deep convection in the tropical Atlantic, Hohenegger and Stevens (2013) suggested that low-level moisture convergence is more efficient than midlevel moistening from detraining cumuli in initiating deep convective cells. Using satellite data, they showed that the presence of congestus clouds does not increase the likelihood for deep convection. The timescale of congestus moistening proceeds too slowly (10 h) to explain the rapid transition from congestus to cumulonimbus $(2-4 \mathrm{~h})$ measured. Also, the observed transition time from congestus to cumulonimbus is shorter over land than the ocean, which suggests that forced ascent induced by differential diurnal heating of the land surface promotes deep convection. Their large eddy simulations showed that the inclusion of parameterized large-scale ascent accelerates the transition by $40 \%$. Using data collected during the Global Atmospheric Research Program Atlantic Tropical Experiment (GATE), they calculated that even very weak large-scale ascent $\left(w=0.01 \mathrm{~ms}^{-1}\right)$ is twice as efficient at triggering deep convection than pure congestus moistening.

The magnitude of surface convergence regulates the vertical extent of convective development. In their idealized numerical simulations on the transition from shallow to deep convection, Rieck et al. (2014) found that convergence of breeze fronts, induced by heterogenous surface sensible and latent heat fluxes, led to a faster transition and enhancements in maximum cloud size, domain vertical velocity, and precipitation rate, when compared to the experiment with horizontally homogeneous surface conditions. In addition, they found that the cloud growth rate was accelerated the most near the time when the opposing fronts collided, further emphasizing the role of convergence in aug- 
menting updraft and convective development. In simulations of deep convection initiation over a mesoscale convergence line, Rousseau-Rizzi et al. (2017) found that magnitude of convergence regulates both the convective-core-size distribution and, in turn, the ultimate height reached by cloud. Stronger convergence generated larger initial cloud horizontal areas at the LFC, which were able to ascend deeper in the troposphere due to their relatively larger buoyancy and vertical velocity during their ascent.

In a case study of convection initiation over the Black Forest mountains of southwest Germany, Bennett et al. (2011) found that upslope flows induced by elevated heating on the mountains created a convergence line that generated stronger convection than that observed over the surrounding plains. In their corresponding numerical simulations, they found that both the first and the heaviest precipitating shallow congestus clouds developed directly above the convergence line. However, the presence of surface convergence was not sufficient to produce the strongest precipitation clouds. Both they and Tompkins (2001) suggested that the initial burst of rainfall moistens air in the inflow regions and creates a subcloud evaporative cold pool. The cold pool can enhance the low-level convergence to provide even stronger low-level forcing for ascent. Klupfel et al. (2012) considered the combination of convergence and positive feedbacks of cold pool as the most important trigger mechanism for their numerical study of mesoscale convective systems in West Africa. Similarly, in their simulations of convection initiation over Amazonia, Khairoutdinov and Randall (2006) found that, despite the complete removal of CIN in the morning, deep convection was still delayed by several hours. Only after cold pools developed from precipitating shallow convection did deep convection initiate. On the contrary, Rousseau-Rizzi et al. (2017) found that core size distribution at the LFC, which was controlled by the magnitude of mesoscale convergence, did not change radically even after the onset of heavy precipitation and the formation of a cold pool. As initial core size was positively correlated to the ultimate height of cloud, they suggested that the mesoscale convergence line outweighed cold pools in regulating the cloud depth.

Recent studies have classified parameters determining the evolution of clouds into "nature" and "nurture" components (Romps and Kuang 2010; Dawe and Austin 2012). Nature refers to initial properties of saturated air observed at cloud base and nurture refers to the subsequent mixing with environmental air during its ascent. Both above-cited studies were devoted to understanding the relative contributions of nature and nurture 
in regulating the dynamics of shallow cumulus clouds. Addressing the problem from the perspective of individual parcels in the cloudy updrafts, Romps and Kuang (2010) found that variability in parcels' properties in the vast majority of the cloud layer correlates well with their net entrainment rates but not with their initial properties near cloud base. In contrast, Dawe and Austin (2012) analyzed the problem by considering the updrafts as an ensemble of cloud entities. Their large eddy simulations showed that while upperlevel cloud thermodynamic properties are determined by entrainment and detrainment events experienced by the cloud as it rises, the horizontal area at cloud base area exerts strong controls over the upper-level area and maximum height of the cloud, because larger clouds undergo less dilution of dry environmental air. Rousseau-Rizzi et al. (2017) suggested that neither nature nor nurture can exclusively determine the depth of deep convection. Varying any one of the environmental parameters (e.g. midlevel moistening by detraining cumuli, cloud-layer environmental lapse rate, background winds) leads to changes in maximum cloud top height. However, they found that the initial core area at cloud base, which is strongly dependent on subcloud forcing, is still the dominant parameter in controlling the depth reached by the convection.

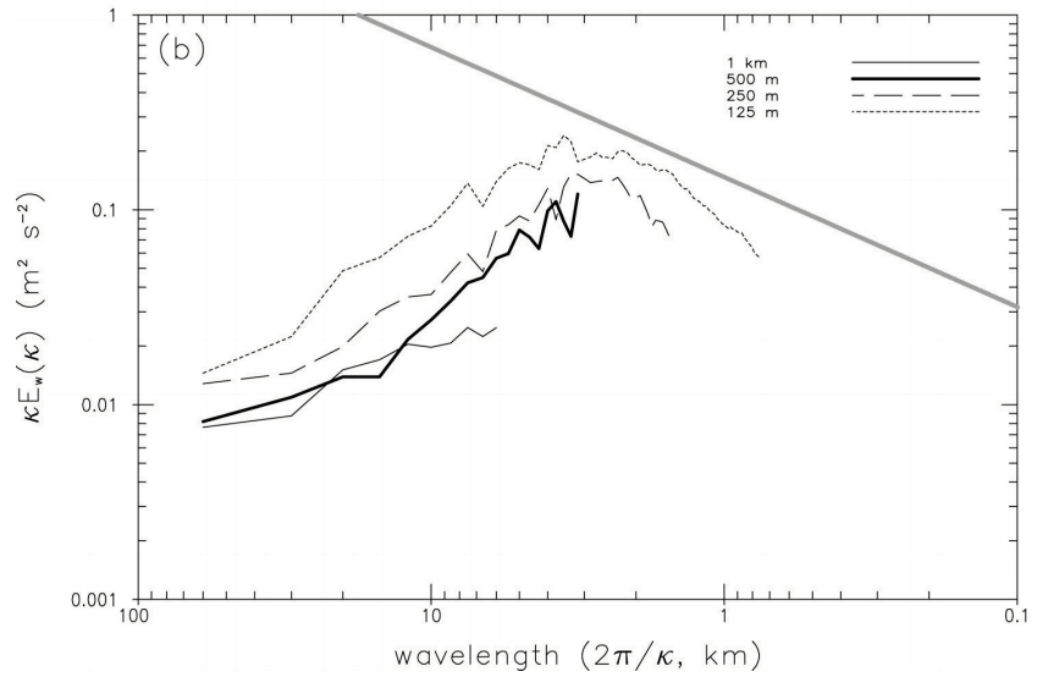

Figure 1: Adapted from Fig. 10 of Bryan et al. (2003), inertial subrange of turbulent eddies are only captured in the $125-\mathrm{m}$ and $250-\mathrm{m}$ simulations.

The numerical representation of moist convection exhibits strong sensitivity to model grid resolution. This sensitivity follows from the diverse scales of turbulent flows that are integral to convective cloud development. These scales range from the synoptic factors regulating the local environment (thousands of $\mathrm{km}$ ), to mesoscale circulations within and 
above the boundary layer, to turbulent and cloud microphysical processes within the clouds ( $\mu \mathrm{m}$ to $\mathrm{mm})$. Simultaneously resolving explicitly all scales of motion including the smallest dissipating eddies is not feasible because it requires large domains with very fine grids that are too computationally expensive for today's computers. Thus, the air motion must be partitioned into resolved-scale and subgrid-scale components. Ideally, the former would include the larger, buoyancy-containing turbulent eddies and the latter would include the inertial subrange, where the forward turbulent cascade is reasonably well understood.

Based on numerical simulations of squall lines, Bryan et al. (2003) suggested that, for adequate resolution, the grid spacing $(\Delta)$ can be much larger than the length scale of the smallest dissipating eddies $(\eta)$ provided that $\Delta$ is much smaller than the length scale $(l)$ of the largest energy-containing eddies, so that the simulated flow is sufficiently turbulent and able to resolve the eddies that carry most of the kinetic energy in the flow. Currently, the horizontal grid spacing of operational weather prediction models $(O(1$ $\mathrm{km}))$ is insufficient to meet these requirements $(O(100 \mathrm{~m}))$. As a result, most turbulence parameterizations, which are designed for the inertial subrange, are unequipped to handle the full range of unresolved processes. This leads to large uncertainties and grid-resolution sensitivities in simulated cloud development.

In simulations of squall lines with horizontal grid spacings ranging from 1 to $12 \mathrm{~km}$, Weisman et al. (1997) found that coarser-grid simulations delayed strengthening of the subcloud cold pool and overpredicted vertical mass transport in the convective system, which led to slower evolution of squall lines. As expected, nonhydrostatic effects on the simulated flow diminished as model grid size increased, because nonhydrostatic effects embedded in small-scaled motions could not be resolved. As the horizontal grid size sets the lower limit of horizonal scale of motion that can be resolved, increasing the grid size causes the resolved flow to become more hydrostatic. Bryan et al. (2003) found that details of squall lines improved significantly as the resolution increased. While systematic trends in changes in precipitation amount, cloud depth, and momentum flux between grid spacings were not identified, the entrainment process and the inertial subrange in onedimensional energy spectra were only captured in the highest resolution (grid spacings of 125- and 250-m) simulations (Fig. 1).

On the other hand, for studies related to deep convection initiation, Waite and 
Khouider (2010) found that increasing horizontal grid size led to reduced dilution of cloudy air and an accelerated transition from shallow congestus to deep convection. Similarly, in a numerical study involving properties of precipitating stratiform clouds using data observed from Tropical Warm Pool-International Cloud Experiment (TWP-ICE), Varble et al. (2014) found an enhancement in both vertical velocity and upward mass flux in convective updraft as grid size increased. However, in another study of numerically simulated deep convection, Hannah (2017) found reductions in vertical mass flux, volume, and the entrainment of convective cores in the cloud layer when grid size increased, suggesting a more complex and possibly case dependent sensitivity of these quantities to grid size.
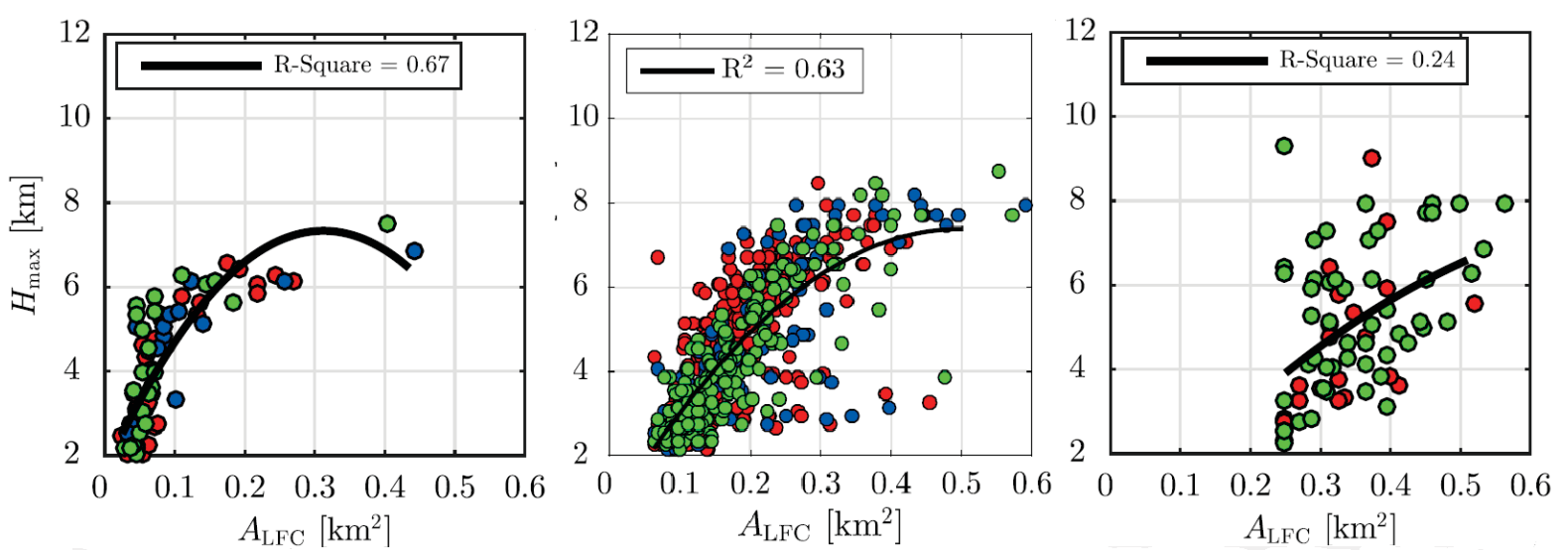

Figure 2: Adapted from Figs. 10 and 16 of Rousseau-Rizzi et al. (2017), scatterplots of final cloud height and initial core at $\Delta=$ (left) $125 \mathrm{~m}$, (middle) $250 \mathrm{~m}$, and (right) $500 \mathrm{~m}$.

In the simulations of Rousseau-Rizzi et al. (2017), decreasing horizontal grid size from $250 \mathrm{~m}$ to $125 \mathrm{~m}$ caused the distribution of initial thermal area at the LFC to shift to smaller sizes (Fig. 2a). This change was associated with a reduction in both cloud-top height and buoyancy realized by the convective thermals. Similarly, increasing horizontal grid size from $250 \mathrm{~m}$ to $500 \mathrm{~m}$ led to increased initial core size (Fig. 2c). However, the cloud-top heights remained similar to those in the 250-m case and the strong correlation between initial core size and cloud-top height weakened.

This study builds on Rousseau-Rizzi et al. (2017) by investigating the sensitivity of simulated convection initiation forced by mesoscale convergence to horizontal grid spacing. The focus is placed on addressing two important objectives: (i) to thoroughly evaluate the sensitivity of simulated deep-convection initiation over a mesoscale convergence to horizontal grid spacing and (ii) to physically interpret the cloud-layer and subcloud 
processes regulating this sensitivity. Section 2 describes the cloud-resolving model used and a general overview of the results. Section 3 presents a detailed physical interpretation of the results. Section 4 presents the conclusions.

\section{Numerical Simulations}

\subsection{Model setup}

Idealized, large-eddy simulations are conducted using CM1 model (release 19.4; Bryan and Fritsch 2002), an Eulerian, nonlinear, compressible, and nonhydrostatic model designed for idealized simulation of moist convection. The model integrates 3D filtered NavierStokes equations using a third-order Runge-Kutta scheme with time-splitting technique to maintain stability of acoustic waves and numerical efficiency (Klemp and Wilhelmson 1978). The model offers a detailed and accurate representation of moist processes in its formulation of governing equations, in particular, the thermodynamic and pressure equations. Along with the inclusion of heat capacity of hydrometers, the equation set nearly conserves both energy and mass, as dissipative heating in the thermodynamic equation and diabatic contributions to the pressure equation are considered.

The current study uses fifth-order advection in both the horizontal and vertical, with positive-definite advection applied to all scalar variables. The Morrison double-moment scheme is used for the parameterization of cloud microphysics with a fixed cloud-droplet concentration of $250 \mathrm{~cm}^{-3}$ (Morrison et al. 2005). A 1.5-order turbulent kinetic energy (TKE) scheme is used for the parameterization of subgrid turbulence. The Coriolis effect is neglected for simplicity, and because the Rossby number of the mesoscale flows of interest is generally large.

The model domain size is $120 \mathrm{~km}$ in the $x$-direction, $180 \mathrm{~km}$ in the $y$-direction, and $20 \mathrm{~km}$ in the $z$-direction. Simulations are conducted with the same background state and surface forcing, but with the horizontal grid spacing $(\Delta)$ systematically varied from $125 \mathrm{~m}$, to $250 \mathrm{~m}$, to $500 \mathrm{~m}$, and to $1000 \mathrm{~m}$. The vertical grid spacing is $100 \mathrm{~m}$ from the surface to $6 \mathrm{~km}$ and then linearly stretches up to $400 \mathrm{~m}$ at $12 \mathrm{~km}$ in the vertical. The lateral boundaries are periodic in $y$ and open in $x$. A no-slip condition is imposed on the bottom boundary and a Rayleigh damping layer is applied over the uppermost $5 \mathrm{~km}$ of the domain. 


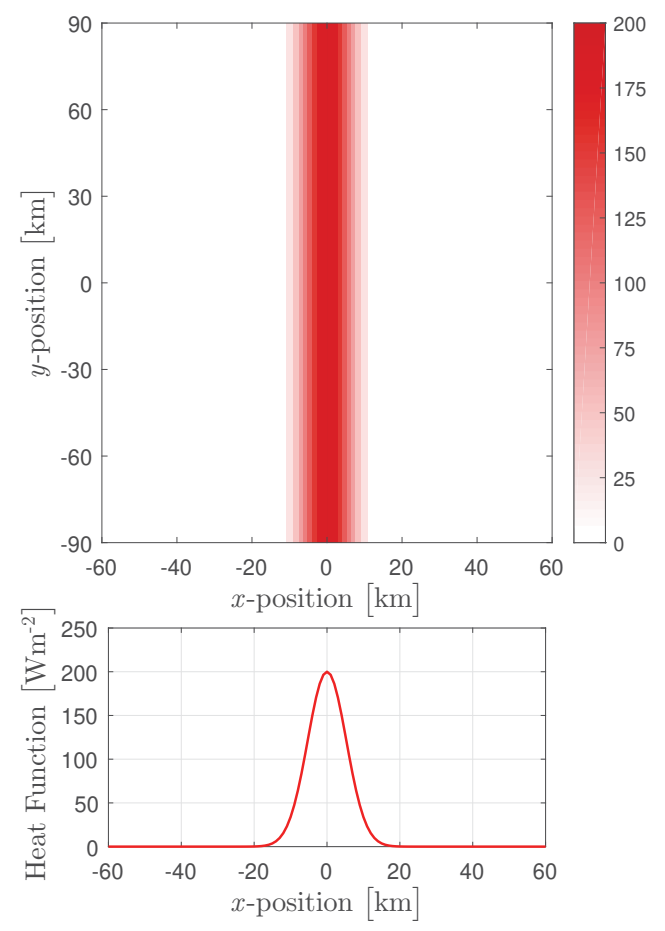

Figure 3: (top) Contours of surface heating function $\left(\mathrm{Wm}^{-2}\right)$ on $x-y$ plane. (bottom) Distribution of Gaussian-shaped surface heat flux across the $x$-dimension.

The main differences between the current simulations and those of Rousseau-Rizzi et al. (2017) are the use of a newer version of CM1 (version 19 rather than version 17) and the inclusion of parcel trajectories. The parcels are initialized at evenly spaced points over the lowest half-level of the staggered model grid, with an inter-parcel spacing of 1 $\mathrm{km}$ in $x$ and $y$. Trajectories of the parcels are updated every time-step, with the parcel output written to file every simulated minute.

Moist convection is initiated over a mesoscale convergence line that is induced by a prescribed surface sensible heat flux (Fig. 3). This flux is specified by the following Gaussian-shaped function:

$$
H(x, t)=H_{0} \exp \left(-\frac{x^{2}}{a^{2}}\right) \sin \Omega t
$$

where $H_{0}=200 \mathrm{Wm}^{-2}, a=7.5 \mathrm{~km}, x$ is zonal distance from the center of the $x$-domain, $t$ is the time from the beginning of the simulation, and $\Omega=2 \pi$ day $^{-1}$ is the diurnal frequency. Hereafter, times are quoted relative to the start of the simulation (e.g., 0600 denotes $6 \mathrm{~h}$ after the onset of the simulation). $H(x, t)$ in (1) is homogeneous in $y$, and gives rise to a mesoscale thermal circulation consisting of two counterrotating vortices in the $x-z$ plane with a combined central updraft that reaches maximum intensity at the 
center of the domain around 6 hours into the simulation (the time of maximum heating in (1). The surface-based convergence induced by the sensible heat flux, and the attendant convective clouds, are confined to the central $20 \mathrm{~km}$ of the domain in all simulations.

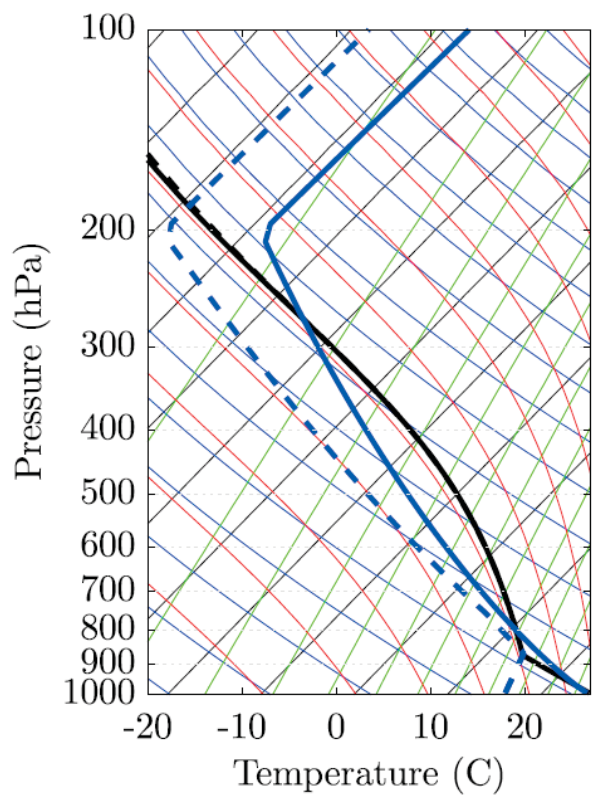

Figure 4: Adapted from Fig. 1b of Rousseau-Rizzi et al. (2017), skew T-log $p$ initial sounding of background temperature (thick blue), background dewpoint (dashed blue), and ascent temperature (thick black).

The simulations are initialized with an environment identical to that in RousseauRizzi et al. (2017) (Fig. 4). Analytic temperature and moisture profiles (Weisman and Klemp 1982) with water vapor mixing ratio of $13 \mathrm{gkg}^{-1}$ in the boundary layer are used. The temperature profile varies from $27^{\circ} \mathrm{C}$ at the surface to $-60^{\circ} \mathrm{C}$ at the tropopause. The sounding constitutes a moderately unstable environment for moist convection. Convective inhibition (CIN) of $50 \mathrm{Jkg}^{-1}$ and convective available potential energy (CAPE) of 1157 $\mathrm{Jkg}^{-1}$ are present at the beginning of the simulation. The large initial CIN prevents cumulus development anywhere except locally along the convergence line. For simplicity, the background wind is zero at all levels, and uniformly distributed random perturbations with a maximum domain-wide magnitude of $1 \mathrm{~K}$ are applied to the potential temperature field to break the horizontal symmetry of the initial state. These perturbations ultimately grow into boundary-layer thermals and cumulus clouds. 


\subsection{Overview of results}
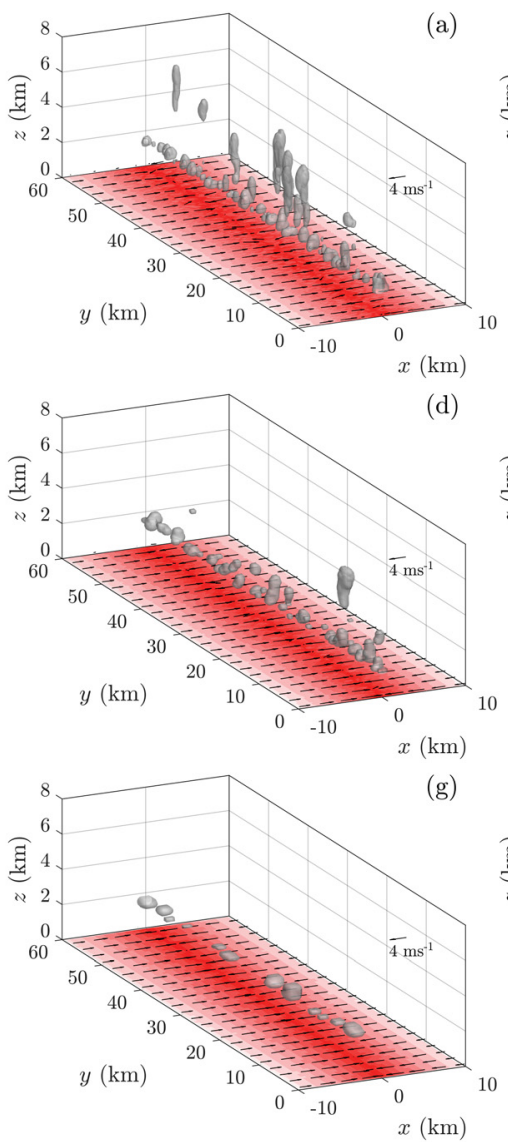
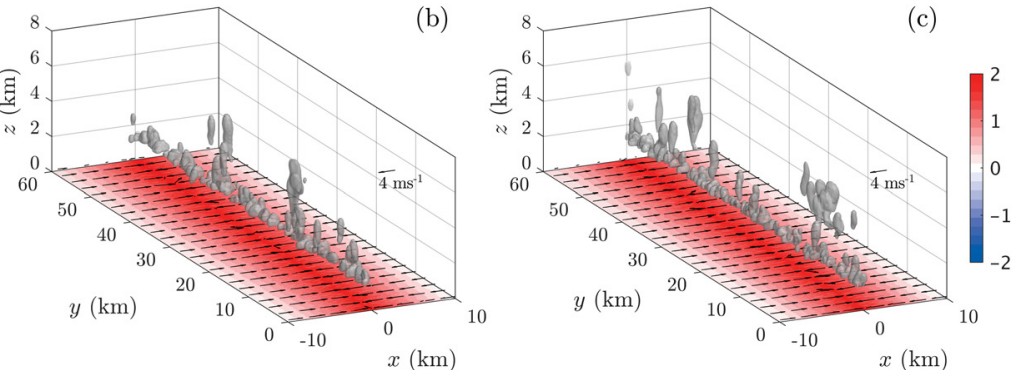

(e)

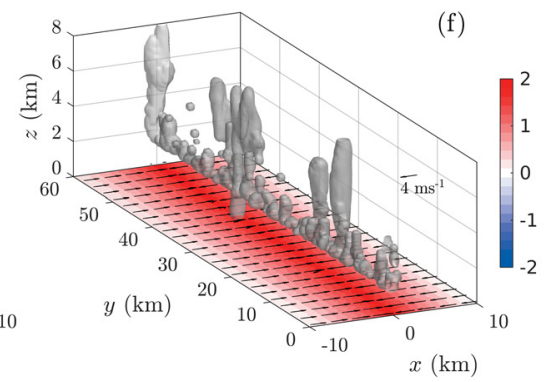

$(\mathrm{h})$

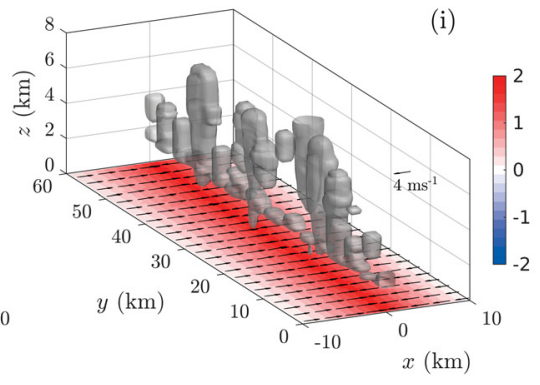

Figure 5: Isosurface (grey contours) of $q_{c}+q_{r}$ equal to $0.1 \mathrm{gkg}^{-1}$ at (a) 04:00, (b) 05:00, and (c) 06:00 for $\Delta=125 \mathrm{~m}$. The same quantities for $\Delta=250 \mathrm{~m}$ are shown in panels (d-f), and for $\Delta=500 \mathrm{~m}$ are shown in panels (g-i). Red contours denote perturbation of potential temperature with respect to mean in $x$-direction on the surface. Black arrows denote surface horizontal wind.

Analysis is focused on results obtained from simulations generated with $\Delta=125 \mathrm{~m}$, $250 \mathrm{~m}$, and $500 \mathrm{~m}$ for brevity, with the 1000-m run used for certain discussions to ensure robustness of tendency drawn from the other three simulations. Fig. 5 presents isosurfaces of cloud water $\left(q_{c}\right)$ and rain water $\left(q_{r}\right)$ mixing ratios, at three different times (04:00, 05:00, and 06:00), for $\Delta=125 \mathrm{~m}$ (Figs. 5a-c), $\Delta=250 \mathrm{~m}$ (Figs. 5d-f), and $\Delta=500 \mathrm{~m}$ (Figs. $5 \mathrm{~g}$ i), respectively. As the magnitude of sensible heat flux increases and destabilizes the air above the heating function, air from each side of the heating function converges toward the centre at roughly $4 \mathrm{~ms}^{-1}$ and eventually collides together at around 04:00. The associated central updraft brings warm thermals to the LFC $(1.75 \mathrm{~km})$ and culminates in the formation of clouds. This boundary layer updraft strengthens with time to produce 
larger clouds that reach greater heights at 05:00. As the magnitude of the sensible heat flux peaks at 06:00, the circulation further intensifies and generates clouds with maximum heights reaching over $8 \mathrm{~km}$. In the remainder of this thesis, we follow Rousseau-Rizzi et al. (2017) by arbitrarily choosing a cloud-top-height threshold of $8 \mathrm{~km}$ to denote "deep" convection. This choice roughly corresponds to the onset of heavy precipitation (rain rates exceeding $10 \mathrm{~mm} \mathrm{hr}^{-1}$ ) at the surface.

Comparing the evolution of cloud fields between the three different horizontal grid spacings, it is evident that the initial growth rate of clouds is the largest in the $125-\mathrm{m}$ simulation and decreases with increasing $\Delta$ at 04:00 (Fig. 5). Clouds as high as $4 \mathrm{~km}$ are simulated in the 125-m run, but the maximum cloud-top height is only around $2 \mathrm{~km}$ in the 250-m and 500-m runs during the same time period. As the boundary layer circulation strengthens over the next hour, the trend reverses: the volume of clouds and maximum cloud-top height decrease as $\Delta$ is reduced. As the circulation strengthens further at 06:00, the differences in cloud development among the three cases are magnified, with the volume and maximum height of clouds substantially larger for the $\Delta=500 \mathrm{~m}$ and $\Delta=250 \mathrm{~m}$ cases than for the $\Delta=125 \mathrm{~m}$.
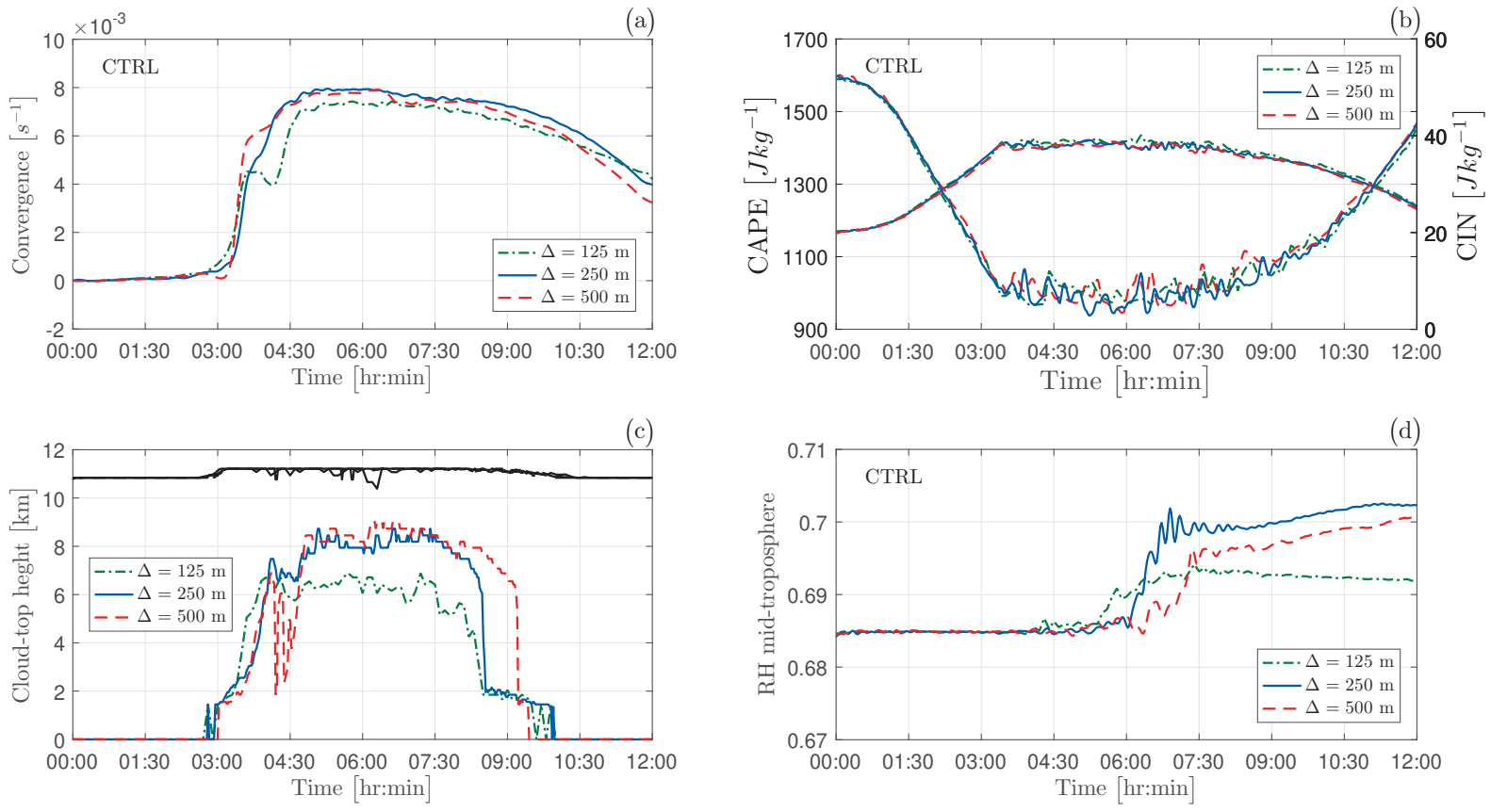

Figure 6: Time series of (a) magnitude of surface convergence, averaged in $y$ along the convergence line and in $x$ over the central $1 \mathrm{~km}$ of the domain; (b) pseudoadiabatic mean-layer (500-m) CAPE and CIN over the central $1 \mathrm{~km}$ of the convergence line; (c) maximum cloudtop height at which $q_{h}=q_{c}+q_{r}+q_{i}+q_{s}+q_{g}$ exceeds $0.1 \mathrm{gkg}^{-1}$ with LNB overlaid in black; and (d) midlevel relative humidity, RH, calculated by volume-weighted average over the central 5 $\mathrm{km}$ and between heights of 3 and $8 \mathrm{~km}$ of the domain. 
Fig. 6a shows the time series of magnitude of surface convergence, which increases slowly up until 03:00. The converging boundary layer flow gives rise to cloudy thermals that fail to reach the LFC (Fig. 6c). Shortly after 03:00, the converging fronts originating along the flanks of the heating function collide at the domain centre, causing an abrupt increase in surface convergence. The associated updraft enhancement forces thermals to cross the LFC, and the clouds deepen rapidly from 03:00 to 04:00. Surface convergence decreases briefly afterwards and so does maximum cloud-top height, likely due to evaporative cooling from precipitation in stabilizing the air near the surface. Although cold pools develop beneath the precipitating clouds (not shown), their lateral propagation causes horizontal convergence to shift away from the central updraft. The ascent associated with these cold pools, however, is insufficient to initiate new convection. Due to continued surface heating, the surface convergence and maximum cloud-top height recover quickly thereafter. As convergence peaks at around 0600 and decreases gradually afterwards, cloud-top height attains its maximum at around 06:30 and plateaus until 07:30. Because the cloud development after this time is not relevant to the convection initiation question, we do not attempt to explain the resolution sensitivities of it.

The sensitivity of surface convergence to $\Delta$ shows that the $125-\mathrm{m}$ run has slightly weaker ( $\sim 5-10 \%$ less) surface convergence for the majority of time than the $250-\mathrm{m}$ and 500-m runs. With the 250-m case having the strongest convergence, the difference between the 250-m and 500-m runs appears to be subtle, when compared with the reduction observed in the 125-m simulation.

The cloud-top height displays the strongest sensitivity to horizontal grid spacing among all the quantities depicted in Fig. 6. For most of the day, the convection in the $125-\mathrm{m}$ case is remarkably $(\sim 2 \mathrm{~km})$ shallower than that in the $250-\mathrm{m}$ and $500-\mathrm{m}$ cases. This obvious sensitivity of cloud depth to $\Delta$ is consistent with Rosseau-Rizzi et al. (2017) and will be analyzed in detail throughout the remainder of this thesis. In addition, the decrease in cloud depth appears qualitatively more pronounced than the weakening in surface convergence, suggesting that there could be processes beyond simply the surface convergence along the domain centerline that regulate the intensity of convective development in our simulations.

Other secondary sensitivities of cloud-top height to $\Delta$ include the rather deep clouds generated in the 125-m run early in the simulation, which corresponds to the relatively 
high surface convergence measured within the same time period. This is most likely because rapidly growing convective thermals develop more readily at higher resolution, due to their improved numerical representation. However, the sensitivity is only transient and eliminated as soon as the converging flow collides at the domain centre.

The convection parameters CAPE and CIN display similar behavior at the three different horizontal grid spacings (Fig. 6b). As CAPE and CIN vary, the value of cloudtop height changes accordingly. When CAPE (CIN) reaches its maximum (minimum) at 06:00, cloud-top height also attains its maximum soon thereafter.

The time series of mid-tropospheric relative humidity $(\mathrm{RH})$ increases due to detrainment by cumuli, particularly after 06:00 when the updraft is the strongest (Fig. 6d). The enhancement in $\mathrm{RH}$ is the highest in the 250-m run and decreases as $\Delta$ varies, which implies that the clouds in the 250-m run exhibit the greatest collective midlevel detrainment among all grid spacings. The increase in $\mathrm{RH}$ is larger in the 500-m run than in the 125-m run, but the most abrupt enhancement in $\mathrm{RH}$ occurs before 06:00 in the 125-m run and after 06:30 in the 500-m run. This is consistent with the more rapid vertical development of clouds in the 125-m case and the slower development in the 500-m case. 


\section{Physical Interpretation of Results}

Given the sensitivities of cloud-top height and surface convergence to horizontal grid spacing $(\Delta)$ documented in Chapter 2, processes that cause the differences observed are investigated here. We begin by evaluating the response of moist thermals in the cloud layer when $\Delta$ is varied. Unless stated otherwise, all subsequent analysis is averaged in time from 05:00 to 06:00, when widespread deep convection initiates within the domain (Fig. 5).

\subsection{Cloud-layer analysis}

A comparison of properties of convective cores within the cloud-layer is shown in Fig. 7. Convective cores are defined as grid points having positive vertical velocity $(w)$, buoyancy $(b)$, and sum of cloud water $\left(q_{c}\right)$ and cloud ice $\left(q_{i}\right)$ mixing ratios greater than $0.1 \mathrm{gkg}^{-1}$.

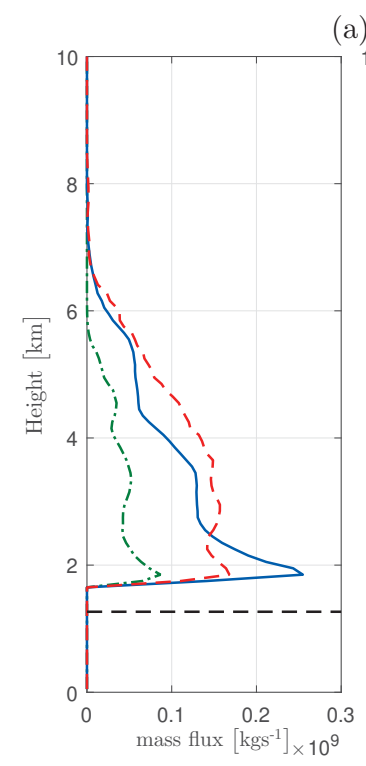

(a)

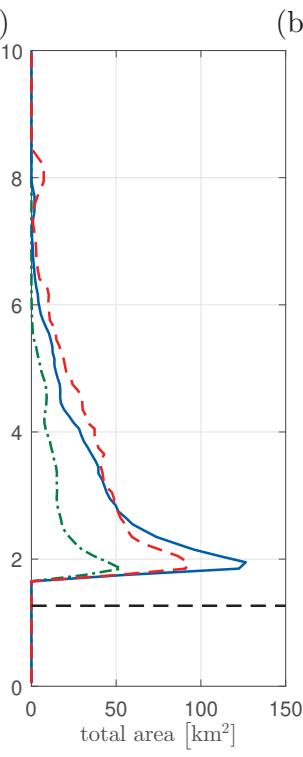

(b)

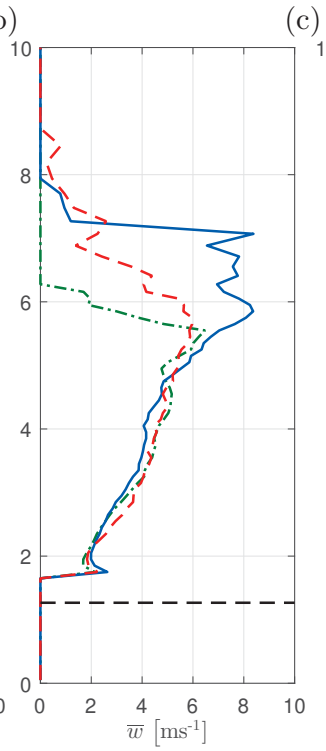

(c)

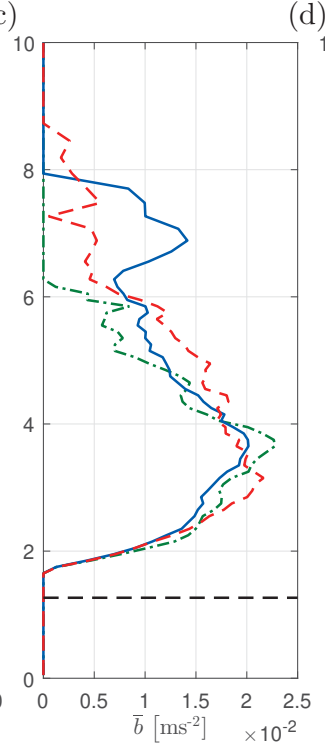

(d)

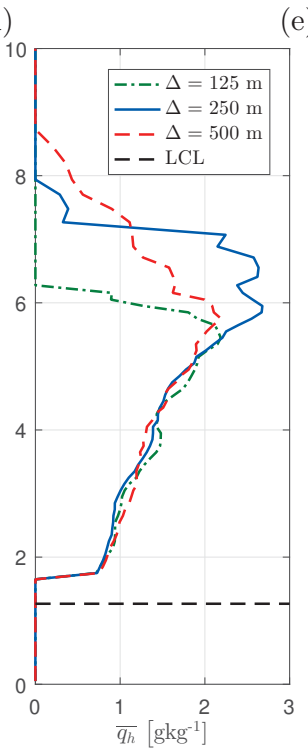

Figure 7: Profiles of (a) total core mass flux, (b) total area, (c) average vertical velocity $\bar{w}$, (d) average buoyancy $\bar{b}$, and (e) average hydrometero mixing ratio $\overline{q_{h}}$, of cores over the whole domain from 05:00 to 06:00. LCL is indicated by dashed black lines.

Profiles of total vertical mass flux of convective cores display significant variations in both magnitude and vertical gradient among the three cases (Fig. 7a). The magnitudes of these profiles become nonzero at the LFC $(1.75 \mathrm{~km})$, above which, the $250-\mathrm{m}$ case has the strongest vertical core mass flux, which is more than $40 \%$ greater than that in the 500-m case and more than twice as large as that in the 125-m run. This suggests that 
the updraft at cloud base, which is primarily driven by subcloud forcing, is the strongest in the $250-\mathrm{m}$ run and weakens as $\Delta$ is either increased or decreased.

The vertical core mass flux $\left(M_{c}\right)$ diminishes with height in all three cases but at different rates. While both $125-\mathrm{m}$ and $500-\mathrm{m}$ runs show similar vertical gradients in $M_{c}$, the 250-m case exhibits the fastest reduction in core mass flux, such that its profile intersects the $500-\mathrm{m} M_{c}$ profile at around $2.5 \mathrm{~km}$ and decreases further aloft. The height at which $M_{c}$ vanishes also depends on horizontal grid spacing, with $M_{c}$ reducing to zero below $6 \mathrm{~km}$ in the $125-\mathrm{m}$ run and at around $8 \mathrm{~km}$ in the other two cases.

The relation between core-averaged vertical velocity $(\bar{w})$, horizontal area of convective core $(A)$, and vertical core mass flux $\left(M_{c}\right)$ at a given height can be estimated by,

$$
M_{c}=\overline{\rho w} A
$$

where $\bar{\rho}$ is the core-averaged density. From Fig. 7b, the variations in total core area closely follow the development of core mass flux, but the differences in core-averaged vertical velocity $(\bar{w})$ across different horizontal gird spacings are relatively limited for altitudes below $6 \mathrm{~km}$ (Fig. 7c). This implies that changes in horizontal core area, as opposed to vertical velocity, regulates the differences in vertical core mass flux within the cloud layer.

Large variations in $\bar{w}$ become apparent near cloud top. Both the 125-m and 500-m runs show reduced $\bar{w}$ above $6 \mathrm{~km}$, with the magnitude of $\bar{w}$ decreasing much more rapidly in the $125-\mathrm{m}$ case. In constrast, the $250-\mathrm{m}$ simulation exhibits further enhancement in $\bar{w}$ over $6 \mathrm{~km}$ up until around $7 \mathrm{~km}$, above which $\bar{w}$ decreases quickly.

Profiles of core-averaged buoyancy $(\bar{b})$ and total hydrometeor mixing ratio $\left(\overline{q_{h}}\right)$ resemble the differences in $\bar{w}$ (Figs. $7 \mathrm{~d}-\mathrm{e}$ ). Although the 500-m run shows slightly larger $\bar{b}$ below $6 \mathrm{~km}$ than the other two cases, varying horizontal grid spacing has modest impact on $\bar{b}$ and $\overline{q_{h}}$ below $6 \mathrm{~km}$, where the majority of the differences in $\bar{b}$ and $\overline{q_{h}}$ unfold. In the 250-m simulation, $\bar{b}$ and $\overline{q_{h}}$ continue to increase above that height, whereas the two quantities decrease above $6 \mathrm{~km}$ in the 125-m and 500-m runs, with the former decreasing much faster than the latter case as cloud top in the 125-m run is substantially lower when compared to its low-resolution counterparts.

The change in vertical core mass flux with height are dependent on the mass entrainment $(E)$ and detrainment rates $(D)$ by, 


$$
\frac{d M_{c}}{d z}=E-D
$$

Entrainment in (3) refers the transition of air from the environment to the volume enclosed by the convective core, while detrainment is defined as the reverse. When there is a net outflow of air mass from the core to the environment, vertical core mass flux decreases with height, and ultimately dissipates at cloud top. As the rate at which the core mass flux decreases with height differs between the three cases, the more negative vertical gradient $\left(d M_{c} / d z\right)$ in the 250 -m simulation could be caused by a relatively low $E$ or high $D$ value associated with the $M_{c}$ profile. Taking into account the differences in core mass flux when evaluating how entrainment and detrainment vary with horizontal grid spacing, (3) is divided by $M_{c}$ and reduced to

$$
\frac{1}{M_{c}} \frac{d M_{c}}{d z}=\epsilon-\delta
$$

where $\epsilon=E / M_{c}$ denotes the fractional entrainment rate and $\delta=D / M_{c}$ denotes the fractional detrainment rate. Using the bulk-plume formulation of Betts (1975), $\epsilon$ is diagnosed by

$$
\frac{d \overline{\phi_{c}}}{d z}=-\bar{\epsilon}\left(\overline{\phi_{c}}-\overline{\phi_{e}}\right)
$$

where $\phi$ is a generic conserved variable, $\overline{\phi_{c}}=\overline{\phi_{c}}(z, t)$ represents the conditional average of the conserved variable within the convective cores at each vertical level, and $\overline{\phi_{e}}=\overline{\phi_{e}}(z, t)$ is the horizontally averaged $\phi$ of the domain at each vertical level. Combining (4) and (5), the fractional detrainment rate $(\bar{\delta})$ is obtained.

As ice often develops in deep convective clouds, ice-liquid water potential temperature $\left(\theta_{i l}\right)$, which is described by Eq. (23) of Bryan and Fritsch (2004), is selected for $\phi$ in (5). This variable is conserved in moist adiabatic processes that include phase changes of both ice and liquid water.

From Fig. 8a, the three cases show similar profiles of $\bar{\epsilon}$ below $5 \mathrm{~km}$. The magnitude of $\bar{\epsilon}$ decreases sharply from its maximum of roughly $5 \times 10^{-3} \mathrm{~m}^{-1}$ at the LFC to around $1 \times 10^{-3} \mathrm{~m}^{-1}$ at $2 \mathrm{~km}$, then it steadily reduces to almost zero by $5 \mathrm{~km}$. Within the heights of $2-4 \mathrm{~km}, \bar{\epsilon}$ appears to be slightly larger in the $250-\mathrm{m}$ simulation, which is consistent with its marginally smaller values of $\bar{w}, \bar{b}$, and $\overline{q_{h}}$ over this layer (Figs. 7c-e). Above 5 $\mathrm{km}$, large variations in $\bar{\epsilon}$ emerge between different horizontal grid spacings. The profile 
(a)

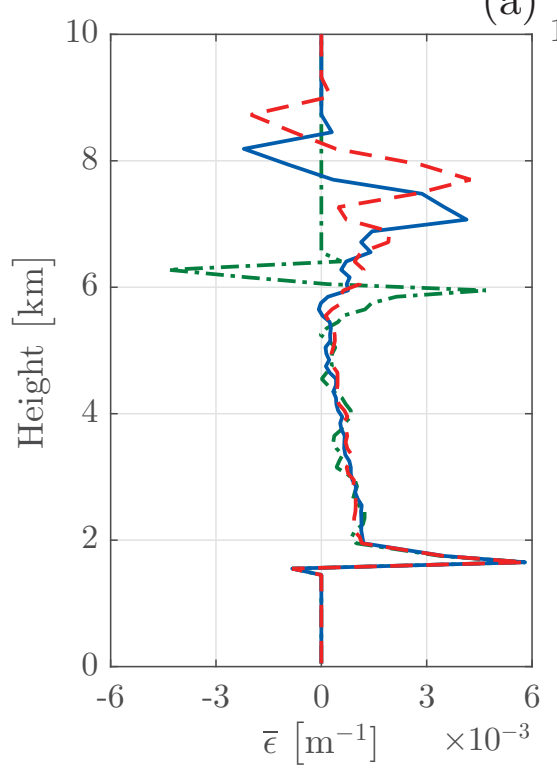

(b)

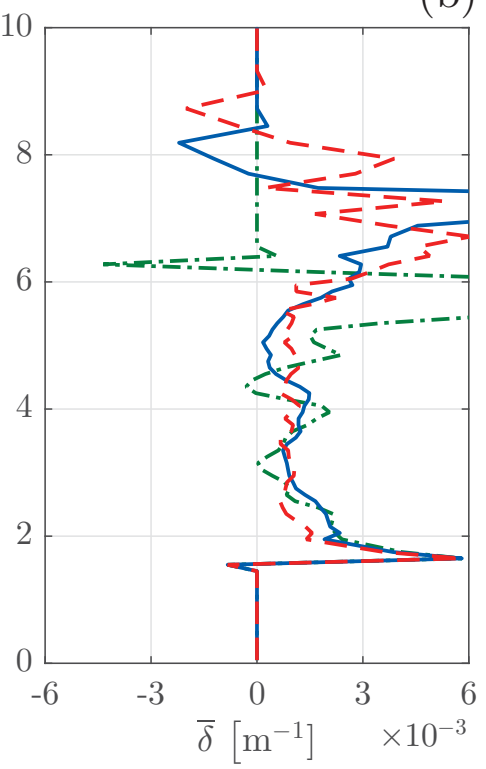

Figure 8: For control case, profile of (a) fractional entrainment rate $\bar{\epsilon}$ and (b) fractional detrainment rate $\bar{\delta}$. The quantities are averaged over number of convective cores from 05:00 to 06:00. Results obtained from simulation with horizontal grid spacing $\Delta$ equal to $125 \mathrm{~m}$ are shown in dash-dot green lines, those obtained with $\Delta=250 \mathrm{~m}$ are shown in solid blue lines, and those with $\Delta$ equal to $500 \mathrm{~m}$ are shown in dashed red lines.

of $\bar{\epsilon}$ oscillates between positive and negative values in each case as it gets closer to cloud top. The fluctuation is congruous to the variations in cloud-top heights shown in Fig. 7c, in that it is the lowest in the 125 -m case and increases with horizontal gird spacing.

Similarly, the detrainment profiles in Fig. 8b begin with local maxima near the LFC, then decrease continuously over the $2-5 \mathrm{~km}$ layer. Above that, $\bar{\delta}$ in each case increases drastically near cloud top. The value of $\bar{\delta}$ exceeds $\bar{\epsilon}$ at most vertical levels, which explains the negative vertical gradient in core mass flux in Fig. 7a. The maximum $\bar{\delta}$ is found at around $6 \mathrm{~km}$ in the $125-\mathrm{m}$ case and $7-8 \mathrm{~km}$ in $250-\mathrm{m}$ and $500-\mathrm{m}$ runs, which corresponds to the cloud-top height in each case. More importantly, $\bar{\delta}$ in the $500-\mathrm{m}$ run is smaller than that in the other two cases from the LFC up to around $4.5 \mathrm{~km}$. This is consistent with the rather minimal decay in core mass flux with height within the same layer in the 500-m run than in the other two cases (Fig. 7a).

The above cloud-layer analysis demonstrates that vertical core mass flux and cloudtop height differ remarkably when horizontal grid spacing changes, with the 125-m run displaying the smallest core mass flux and the shallowest cloud development. However, differences in the conditionally averaged core properties $\left(\bar{w}, \bar{b}, \overline{q_{h}}, \bar{\epsilon}\right.$, and $\left.\bar{\delta}\right)$ between the three cases are fairly limited, except in the vicinity of cloud top. This suggests that 
the ultimate height of cloud is primarily determined by the intensity of core mass flux, particularly near cloud base, which is driven by subcloud updraft reaching the corebearing layer from below.

\subsection{Parcel trajectories}

Insight into the role of subcloud forcing in regulating convection in the cloud layer is provided by parcel trajectory analysis. This section examines how the motion of subcloud parcels driven by the central convergence line differs when horizontal grid spacing is varied. Specifically, does the likelihood of subcloud parcels reaching the cloud layer and higher altitudes change with horizontal grid spacing?

A volume defined as the "central convergence region" (CCR), which encloses the central $1 \mathrm{~km}$ in $x$, the entire $y$-domain, and the layer from the surface to the $y$-averaged LCL in $z$, is used to facilitate the analysis of forward trajectories of parcels. Over the course of the simulation, the number of parcels flowing into the CCR is measured and their evolution with time are recorded.

\begin{tabular}{cccc}
\hline & $\Delta=125 \mathrm{~m}$ & $\Delta=250 \mathrm{~m}$ & $\Delta=500 \mathrm{~m}$ \\
\hline $\mathrm{N}$ & 14422 & 14929 & 15113 \\
$\mathrm{~N}_{\mathrm{LCL}}$ & $1427(10 \%)$ & $2551(17 \%)$ & $1946(13 \%)$ \\
$\mathrm{N}_{\text {core }}$ & $77(0.5 \%)$ & $243(1.6 \%)$ & $219(1.4 \%)$ \\
\hline
\end{tabular}

Table 1: Statistics of parcel-trajectory analyses in the 125-m, 250-m, and 500-m runs. In the left column, $\mathrm{N}$ is the total number of parcels entering the central converging region (CCR), $\mathrm{N}_{\mathrm{LCL}}$ is the number of parcels entering the CCR that reach the LCL, and $\mathrm{N}_{\text {core }}$ is the number of parcels that cross the LFC to become a convective core.

From Table 1, a total of $\mathrm{N}=14000-15000$ parcels are found to enter the CCR in the three cases, with the 125-m run having the fewest, which is consistent with its relatively weak surface convergence (Fig. 6). Among these parcels, only 10-20\% of them are able to cross the LCL, with the smallest parcel fraction in the 125-m run and the largest parcel fraction in the 250-m run. These differences are consistent with the distribution of vertical velocity of parcels near the LCL $\left(w_{\mathrm{LCL}}\right)$ shown in Fig. 9a, where the mean $w_{\mathrm{LCL}}$ is the lowest in the $125-\mathrm{m}$ run and shifts toward larger values in the $500-\mathrm{m}$ and $250-\mathrm{m}$ runs. In all three cases, only a tiny fraction (approximately $1 \%$ ) of the parcels entering the CCR eventually become positively buoyant and become convective cores. Again, the 
parcel fraction $\left(N_{\text {core }}\right.$ in Table 1$)$ is the smallest in the $125-\mathrm{m}$ run and the largest in the 250-m run.

Both the distribution of $w_{\mathrm{LCL}}$ in Fig. 9a and the core mass flux profile in Fig. 7a suggest that subcloud ascent is the strongest in the $250-\mathrm{m}$ run and the weakest in the 125-m run. Subcloud parcels with larger $w_{\mathrm{LCL}}$ are more likely to overcome the negative buoyancy before reaching the LFC, which enables the 250-m run to have the highest parcel fraction $\left(N_{\text {core }}\right)$ in Table 1 and the strongest core mass flux near cloud base in Fig. 7a. Although the subcloud forcing for ascent is weaker in the 500-m run than in the 250-m run, higher percentages of the parcels that ever entered the CCR in the 500-m case are found at all vertical levels above the LFC (Fig. 9b). This can be attributable to the relatively small fractional detrainment rate $(\delta)$ at midlevels $(2-4 \mathrm{~km})$ associated in this case, allowing the subcloud parcels to remain longer within the ascending cores as they ascend. In contrast, the parcels in the 125-m run are subject to weaker subcloud forcing and higher fractional detrainment rate, leaving them with the lowest core mass flux in Fig. $7 \mathrm{a}$ and the fastest cloud dissipation at lower heights in Fig. 9b.

(a)
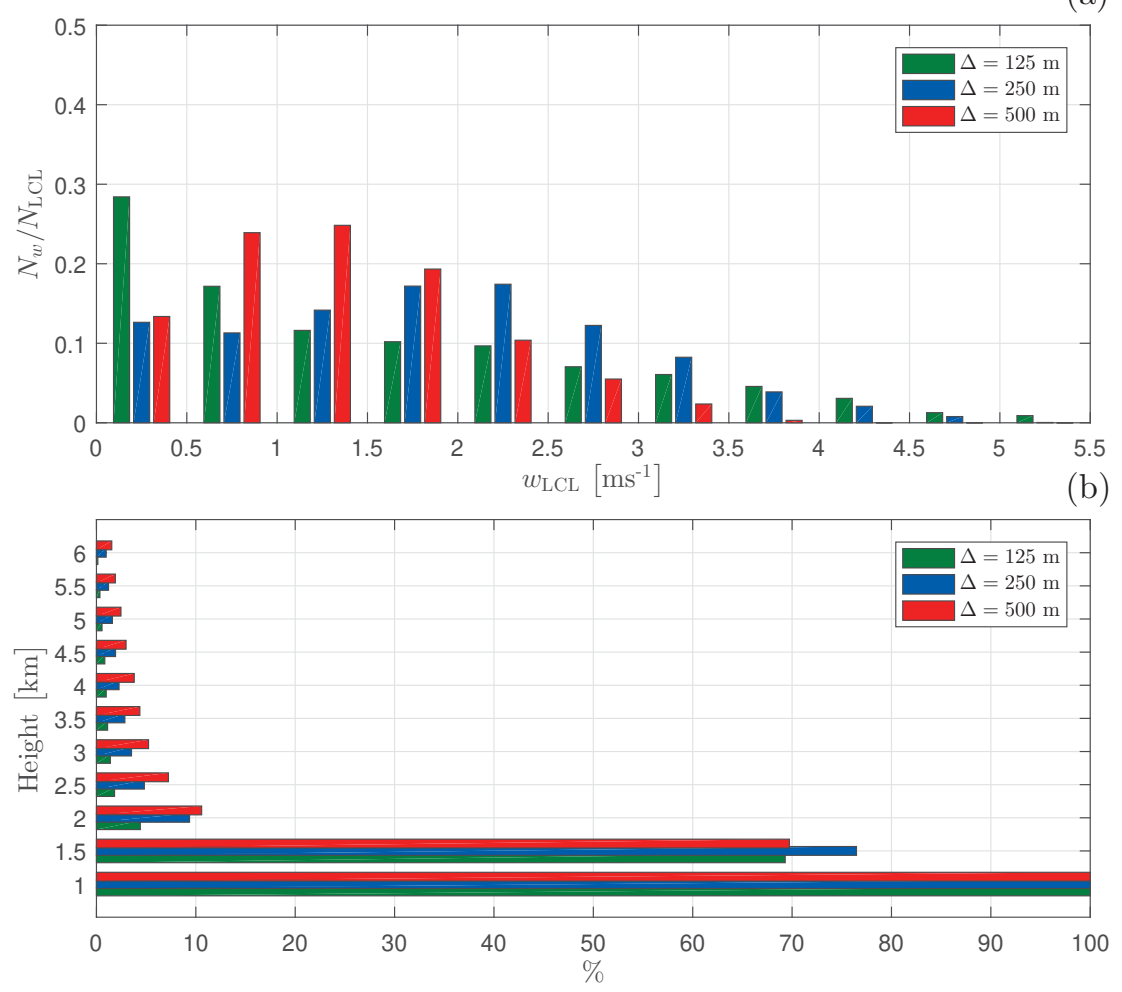

Figure 9: (a) Distribution of vertical velocity $w_{\text {LCL }}$ of parcels prior to crossing the LCL for the last time. The number of parcels in each velocity bin is $\mathrm{N}_{\mathrm{w}}$ and the total number of parcels reaching the LCL in each simulation is $\mathrm{N}_{\mathrm{LCL}}$. (b) Percentage (\%) of parcels having maximum height greater than or equal to the value denoted by each bin on the $y$-axis, among the subset of parcels that ever entered the CCR and reached the LCL 


\subsection{Subcloud-layer analysis}

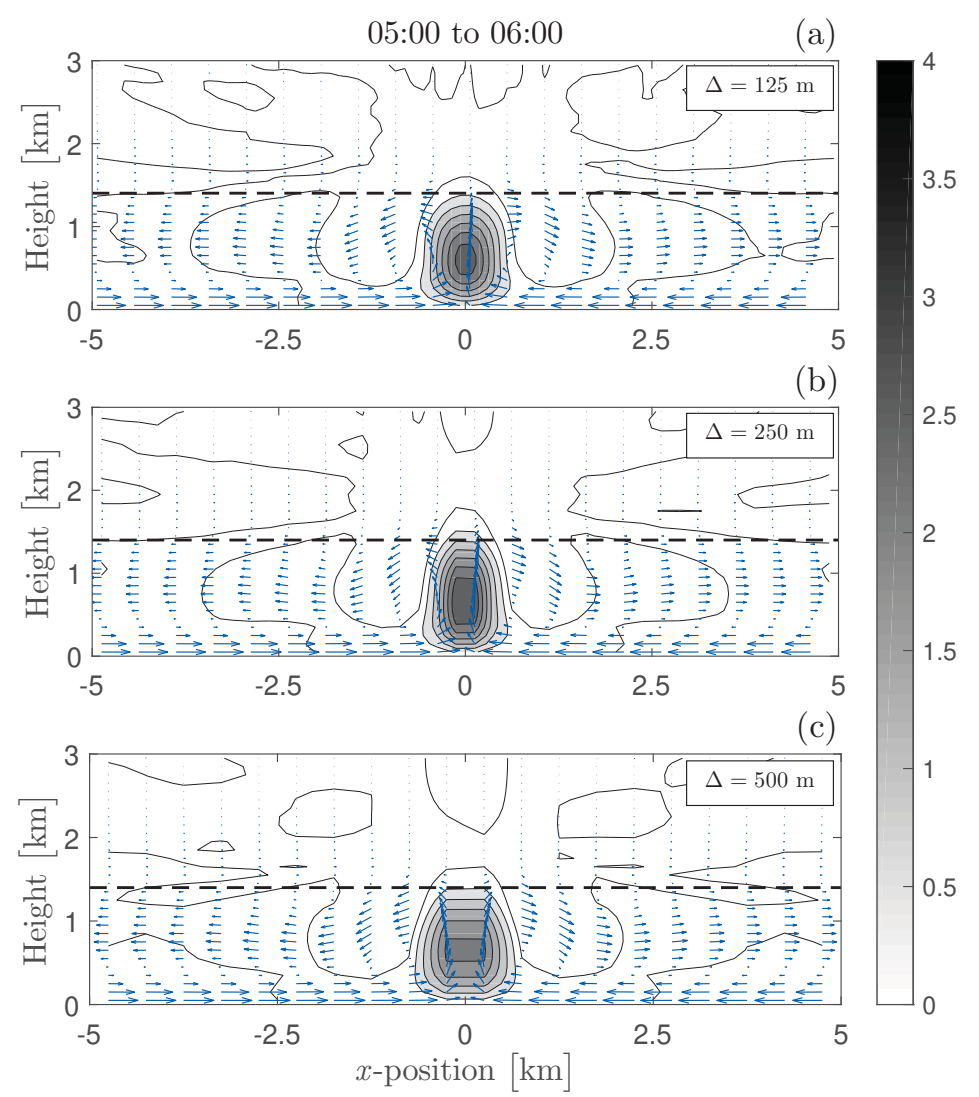

Figure 10: Contours of $y$-averaged $w$ (filled with greyscale contours) and $y$-averaged wind vectors (blue arrows) over the time interval stated from simulation with grid-spacing of (a) $\Delta=125 \mathrm{~m}$, (b) $\Delta=250 \mathrm{~m}$, and (c) $\Delta=500 \mathrm{~m}$. LCL is indicated by dashed black lines. Contour lines range from $0 \mathrm{~ms}^{-1}$ to $4 \mathrm{~ms}^{-1}$ with $0.5 \mathrm{~ms}^{-1}$ increments.

The findings thus far indicate that the cloud-top height is highly dependent on vertical core mass flux, which at cloud base is governed by the strength of subcloud updraft in bringing parcels into the cloud layer. To interpret the role of subcloud processes in governing the updraft strength and their sensitivity to $\Delta$, we compare the $y$-averaged thermal circulation around $x=0 \mathrm{~km}$ in Fig. 10. The three cases exhibit similar circulation that is characterized by a strong central updraft stretching from the domain center up to the LCL, with a shallow layer of horizontal convergence below the updraft and a deeper layer of divergence aloft.

The differences in Fig. 10 may seem subtle, yet they still merit some attention. The maximum $y$-averaged $w$ is the largest in the 250-m case and decreases as resolution varies, with the smallest value found in the 500-m case. More importantly, the magnitude of $\bar{w}$ at the LCL, as shown by the black contour lines, is also the highest in the 250-m case 
and decreases in the other two cases. This is consistent with the median $w_{\mathrm{LCL}}$ being the largest in the 250-m run and the weakest in the 125-m run, as shown in Fig. 9a. Although the 500-m run displays the weakest $y$-averaged $w$ field, the magnitude decreases the least rapidly with height among the three cases, which makes the median $w_{\mathrm{LCL}}$, hence subcloud forcing for ascent, fall in-between the 125-m and 250-m cases.

Overall, the updraft in the 250-m run is the narrowest, deepest, and strongest. The updraft in the $125-\mathrm{m}$ run is wilder, shallower, and weaker than the 250-m run. Among the three cases, the updraft in the 500-m run is the widest and weakest, but with the slowest decay in amplitude with height.

\subsubsection{The $w$ budget}

The sensitivity of subcloud dynamics to horizontal grid spacing is interpreted through the $w$ budget. The governing equation for vertical velocity $(w)$ in the CM1 model is given as follows

$$
\frac{\partial w}{\partial t}=-\frac{1}{\rho_{0}}\left[\nabla \cdot\left(\rho_{0} \mathbf{u} w\right)-w \nabla \cdot\left(\rho_{0} \mathbf{u}\right)\right]-c_{p} \theta_{\rho} \frac{\partial \pi^{\prime}}{\partial z}+g \frac{\theta_{\rho}^{\prime}}{\theta_{\rho 0}}+T_{h, \mathrm{sgs}}+T_{v, \mathrm{sgs}}+D_{h}+D_{v}
$$

where $\mathbf{u}=\mathbf{u}(x, y, z)$ is the wind velocity, $\theta_{\rho}=\theta_{\rho}(x, y, z)$ is the density potential temperature, and $\pi=\pi(x, y, z)$ is the Exner function. In (6), the subscript " 0 " denotes the hydrostatically balanced initial state that varies only in the $z$-direction $\left[\rho_{0}(z), \theta_{\rho 0}(z)\right.$, and $\pi_{0}(z)$, and primes denote perturbations from the initial state.

The terms on the right-hand side (RHS) of (6) are, from left to right, advection (ADV), vertical perturbation pressure gradient (VPPG), buoyancy (BUOY), horizontal $\left(T_{h, \mathrm{sgs}}\right)$ and vertical $\left(T_{v, \text { sgs }}\right)$ subgrid turbulent mixing, and horizontal $\left(D_{h}\right)$ and vertical $\left(D_{v}\right)$ numerical diffusion. The advection term in (6) is formulated as a sum of a flux-form term and a divergence term, which is considered more efficient for momentum conservation (Bryan and Fritsch 2002).

The significance of each term in the $w$ budget over the lowest $3 \mathrm{~km}$ in the 250 -m run is evaluated from the horizontally (over the CCR) and temporally (over 05:00 to 06:00) averaged profiles shown in Fig. 11. The two dominant terms are BUOY and VPPG, with the former being positive below $1 \mathrm{~km}$ and negative between 1-2 km. This is expected as 
heated subcloud thermals penetrate the stably stratified atmosphere above cloud base, leaving $\theta_{\rho}^{\prime}$ negative with respect to the horizontal mean of $\theta_{\rho}$ in the layer. While VPPG generally opposes BUOY, its magnitude is slightly smaller. Other important terms are the horizontal and vertical components of ADV, which alternate from negative (0-700 $\mathrm{m})$ to positive (700-1500 m). The magnitudes of the remaining diffusion and subgrid turbulence terms are negligible compared to BUOY and VPPG.

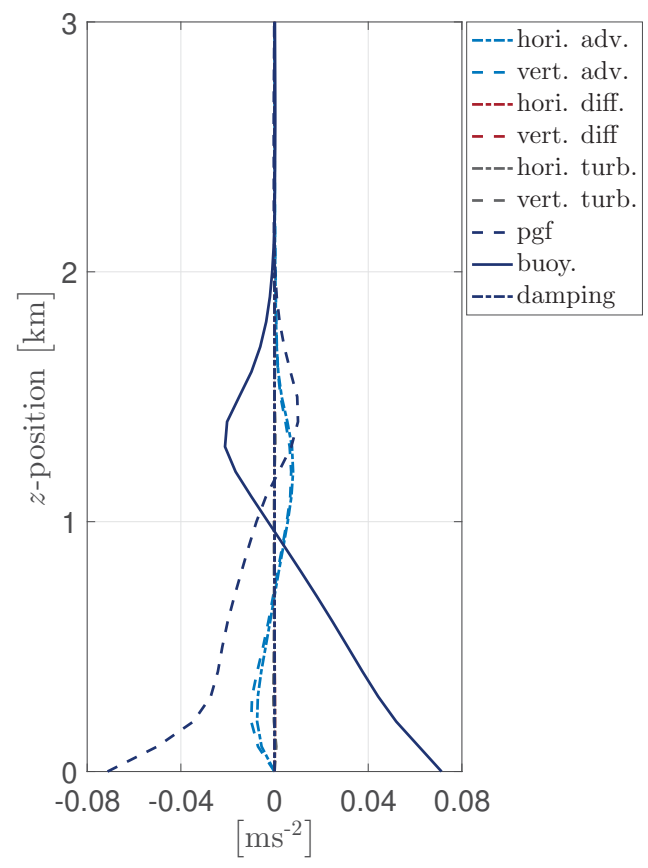

Figure 11: Profile of each term in $w$-budget model output averaged horizontally over the CCR and temporally from 05:00 to 06:00.

Comparison of the sum of RHS terms in (6) and the LHS tendency term (averaged horizontally over the CCR and temporally from 05:00 to 06:00) is given in Fig. 12. As the RHS sum in Fig. 12a is nearly identical to the LHS tendency term in Fig. 12b for each case, the $w$ budget is deemed valid to provide insight into the flow dynamics in the subcloud layer. Over this time period, subcloud forcing for ascent differs when horizontal grid spacing varies, with the weakest forcing found in the 500-m run and stronger forcing displayed in the 125-m and 250-m runs. The height at which maximum forcing occurs is higher in the $250-\mathrm{m}$ run than in the $125-\mathrm{m}$ run. Above $2 \mathrm{~km}$, the forcing is slightly positive for the $250-\mathrm{m}$ and $500-\mathrm{m}$ cases, and slightly negative for the $125-\mathrm{m}$ case.

An estimate of the impact of RHS forcing on altering the updraft magnitude is obtained by integrating the maximum RHS forcing over the hourlong period of time, which gives a change in vertical velocity of roughly $0.1 \mathrm{~ms}^{-1}$. When compared to the maximum 

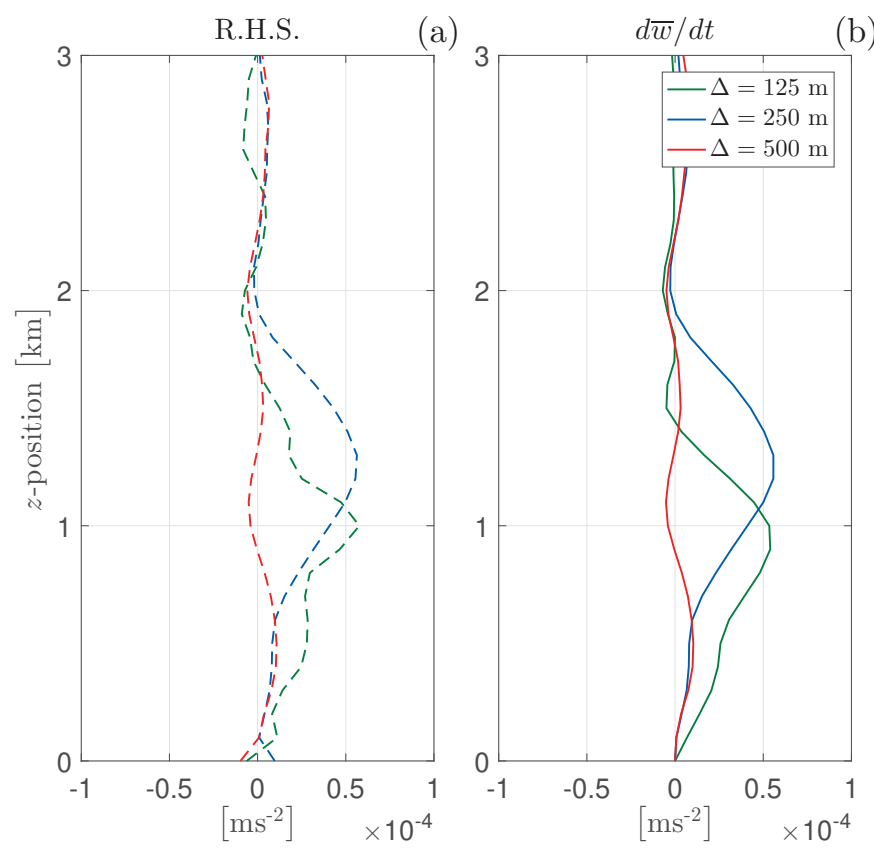

Figure 12: Comparison of (a) the RHS forcing terms of (6) to (b) the LHS time-tendency of (6) between the three cases. The profiles are averaged horizontally over the CCR and temporally over 05:00 to 06:00.

updraft velocity of $4 \mathrm{~ms}^{-1}$ in Fig. 10, the change in $w$ induced by the RHS forcing is negligible, implying that the updraft has already attained quasi-steady state by 05:00. Thus, rather than attemping to explain the negligible variations evident in Fig. 12, subsequent analysis is focused on interpreting the contribution of each RHS term in (6) to the quasi-steady $w$ field.

Given that the $w$ budget is dominated by the BUOY and VPPG terms, these two terms are examined thoroughly to understand their role in governing the subcloud dynamics and their sensitivity to horizontal grid spacing. As shown in Fig. 13b, the distribution of time- and $y$-averaged BUOY in the $250-\mathrm{m}$ case is strongly positive at the surface and decreases with increasing height. At around $z=1 \mathrm{~km}$, its magnitude changes from positive to negative and reaches its local minimum at $x=0 \mathrm{~km}$ and $z \approx 1.3 \mathrm{~km}$. This vertical variation in buoyancy $(b)$ stems from the mesoscale thermal circulation with a central updraft transporting surface-based thermals with positive $\theta_{\rho}^{\prime}$ initially to the stably stratified free troposphere, where $\theta_{\rho}^{\prime}$ becomes negative. 


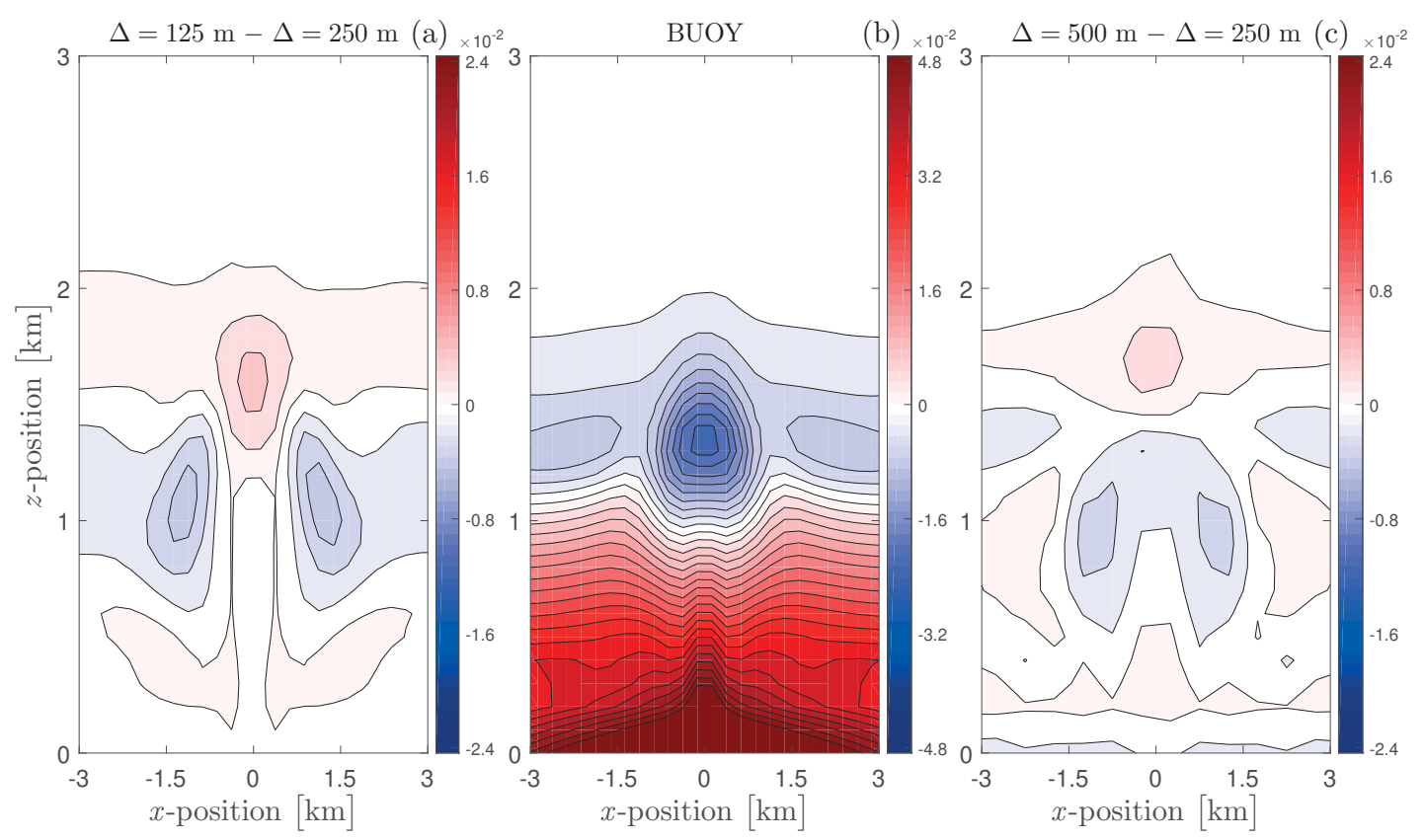

Figure 13: Contours of $y$ - and time-averaged (05:00-06:00) buoyancy term in (6). Panel (b) shows contours of buoyancy term when $\Delta=250 \mathrm{~m}$. Panels (a) and (c) show differences in buoyancy between $\Delta=125 \mathrm{~m}$ and $\Delta=250 \mathrm{~m}$ cases and $\Delta=500 \mathrm{~m}$ and $\Delta=250 \mathrm{~m}$ cases respectively.

The differences in BUOY distribution between the 125-m and 250-m cases and 500-m and 250-m cases are shown in Fig. 13a and Fig. 13c, respectively. Between the 125$\mathrm{m}$ and $250-\mathrm{m}$ cases, larger buoyancy appears above the central updraft at $z \approx 1.6 \mathrm{~km}$ and smaller buoyancy along the sides of the updraft at $z \approx 1 \mathrm{~km}$ and $x= \pm 1.3 \mathrm{~km}$ (Fig. 13a). These variations are caused by a weakening in the thermal circulation in the 125-m run. With fewer thermals penetrating from the subcloud layer into the stably stratified free troposphere, less negative buoyancy is generated. Hence, the $y$-averaged buoyancy becomes larger above the central updraft. With less downdraft descending into the boundary layer from above, the $y$-averaged buoyancy along the sides of the updraft becomes smaller. As the updraft is also weakened in the 500-m run, similar but less pronounced differences in the BUOY distribution appear in Fig. 13c.

In contrast to BUOY, the distribution of VPPG in the 250-m run is strongly negative near the surface and increases with height up to a local maximum at $z \approx 1.5 \mathrm{~km}$ (Fig. 14b). In a hydrostatically balanced atmosphere, the separation of pressure surfaces increases in the presence of low-level warm anomaly, which induces low pressure near the surface and high pressure aloft. Hence, VPPG is directed downward within the boundary layer and upward above it. Also, the local maximum of VPPG at $x=0 \mathrm{~km}$ and $z=1.5$ 
$\mathrm{km}$ is caused by the penetration of central updraft into the troposphere, which generates negative buoyancy and enhances the high pressure above the updraft.

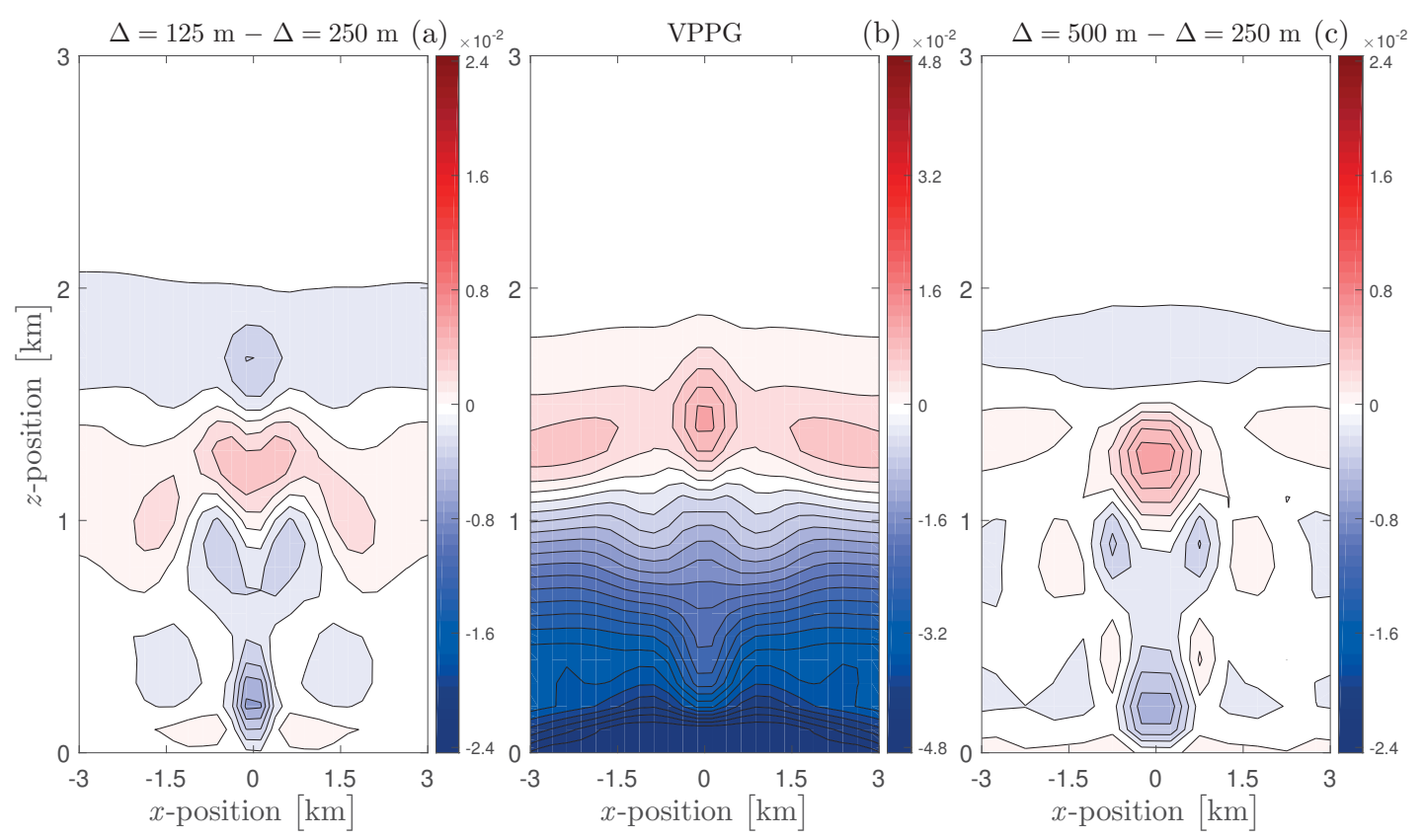

Figure 14: As in Fig. 13, but for VPPG term in (6).

Differences in VPPG distribution between the 125-m and 250-m cases in Fig. 14a are similar to that between the 500-m and 250-m cases in Fig. 14c. Both panels exhibit a strong enhancement of negative VPPG within a shallow region form the surface to $z \approx 0.5 \mathrm{~km}$ along the centerline, above which sits a series of alternating negative and positive differences in VPPG extending over the top of the boundary layer. Although other secondary differences are present along the sides of the updraft, emphasis is placed on interpreting the large-amplitude features at $x=0 \mathrm{~km}$, where the majority of vertical motion takes place.

The alternating differences in VPPG over the layer of $z=1-2 \mathrm{~km}$ in Fig. 14a and Fig. 14c are most likely due to a reduced depth in the central updraft simulated in the 125-m and 500-m cases, which have also been mentioned in Fig. 10. Penetration of the thermally induced updraft into the stably stratified troposphere generates high pressure, which is shifted downward for shallower updrafts. Thus, such positional shifts give rise to alternating positive and negative differences in VPPG.

In contrast to all of the differences discussed thus far, the strongly negative difference in VPPG over the 0-500 m layer along the domain centerline are not associated with any difference in BUOY in Fig. 13a and Fig. 13c. The quantitative impact of this distinctive 
feature in VPPG difference on the updraft strength is estimated by applying a steady, inviscid, and 1D form of (6), which, for simplicity, only considers the VPPG and BUOY terms:

$$
\begin{gathered}
w \frac{\partial w}{\partial z} \approx b-c_{p} \theta_{\rho} \frac{\partial \pi^{\prime}}{\partial z} \\
\frac{1}{2} \frac{\partial}{\partial z}\left(w^{2}\right) \approx b-c_{p} \theta_{\rho} \frac{\partial \pi^{\prime}}{\partial z}
\end{gathered}
$$

The zonal velocity, hence zonal advection, is omitted on the left-hand side (LHS) of (8), because the flow at $x=0 \mathrm{~km}$ is predominantly vertical. In the $250-\mathrm{m}$ run, the LHS of (8) can be approximated by using the upper $\left(\left.w\right|_{500 m} \approx 3 \mathrm{~ms}^{-1}\right.$; Fig. 10b) and lower $\left(\left.w\right|_{\mathrm{sfc}}=0 \mathrm{~ms}^{-1}\right)$ bounds of $w$ within this layer to give

$$
\frac{1}{2} \frac{\left.w^{2}\right|_{500 m}-\left.w^{2}\right|_{\mathrm{sfc}}}{500 m} \approx 9 \times 10^{-3} \mathrm{~ms}^{-2}
$$

To evaluate the impact of the stronger adverse low-level VPPGs in the 125-m and 500-m cases, we subtract from $(9)$ the mean VPPG difference over the $0-500 \mathrm{~m}$ layer $(\approx 0.005$ $\mathrm{ms}^{-2}$; Fig. 14a and Fig. 14c) between the 125-m and 500-m cases and the 250-m cases. With this feature taken into account, the right-hand side of (8) becomes $4 \times 10^{-3} \mathrm{~ms}^{-2}$. Hence, the new $w$ at $z=500 \mathrm{~m}$ is obtained by solving (9) with the adjusted right-hand side to give $\left.w\right|_{500 m}=2 \mathrm{~ms}^{-1}$, which suggests that the enhanced low-level VPPG in the $125-\mathrm{m}$ and $500-\mathrm{m}$ cases relative to the $250-\mathrm{m}$ case leads to a significant $(\sim 33 \%)$ weakening of the central updraft.

\subsubsection{Updraft morphology}

The preceding analysis illustrates that stronger adverse VPPG and weaker vertical velocity are present within the shallower and wider updrafts simulated in the $125-\mathrm{m}$ and 500-m runs (Fig. 10 and Fig. 14). However, the mechanism behind the role of updraft size in regulating updraft velocity has not been discussed explicitly. This inspires a more quantitative assessment of the impact of updraft morphology on perturbation pressure and updraft strength between the three cases.

The dynamics of shallow boundary-layer circulations can be explained by using the Boussinesq form of (6), with subgrid turbulence and numerical diffusion neglected for 
simplicity, which is

$$
\frac{D w}{D t}=-\phi_{z}+b
$$

where $\phi=p^{\prime} / \rho_{00}, p^{\prime}$ is the pressure perturbation from the base state, and $\rho_{00}$ is a constant reference density. With no vertical shear in the simulated background flow, a diagnostic equation for $\phi$ can be linearly approximated as

$$
\nabla^{2} \phi=\nabla^{2} \phi^{b}=b_{z}
$$

where $\phi^{b}$ is called the buoyancy pressure perturbations and can be further partitioned into hydrostatic $\left(\phi^{b h}\right)$ and nonhydrostatic $\left(\phi^{b n}\right)$ components (e.g., Markowski and Richardson 2010). By definition, the former is given by

$$
\phi_{z}^{b h}=b
$$

which reduces (10) to

$$
\frac{D w}{D t}=-\phi_{z}^{b n}
$$

Hence, the vertical acceleration is determined by the nonhydrostatic component of vertical presssure perturbation gradient. Taking the $z$-derivative of (11) for $2 \mathrm{D}(x-z)$ flow gives

$$
\begin{aligned}
\nabla^{2} \phi_{z}^{b h}+\nabla^{2} \phi_{z}^{b n} & =b_{z z} \\
\left(\phi_{z}^{b h}\right)_{x x}+\left(\phi_{z}^{b h}\right)_{z z}+\nabla^{2} \phi_{z}^{b n} & =b_{z z}
\end{aligned}
$$

Substituting (12) into (15) yields the following expression

$$
\begin{aligned}
\nabla^{2} \phi_{z}^{b n} & =-\left(\phi_{z}^{b h}\right)_{x x} \\
& =-b_{x x}
\end{aligned}
$$

Assuming a periodic waveform solution

$$
\left[b, \phi_{z}^{b n}\right]=\left[\hat{b}, \hat{\phi}_{z}^{b n}\right](z) e^{i(k x+m z-w t)},
$$


where $\hat{b}$ and $\hat{\phi}_{z}^{b n}$ are complex amplitudes of $b$ and $\phi_{z}^{b n}, k$ and $m$ are the $x$ and $z$ wavenumbers, and $\omega$ is the angular frequency, we obtain

$$
-\hat{\phi}_{z}^{b n}=\frac{k^{2}}{k^{2}+m^{2}} \hat{b}
$$

Substituting (19) into (13) gives

$$
\frac{D w}{D t}=\frac{k^{2}}{k^{2}+m^{2}} \hat{b}
$$

Using scale analysis, $k \sim L^{-1}$ and $m \sim H^{-1}$, where $L$ and $H$ are horizontal and vertical length scales, (20) may be expressed as

$$
\frac{D w}{D t} \approx \frac{(H / L)^{2}}{1+(H / L)^{2}} \hat{b}
$$

Thus, for a given $b$, the vertical acceleration approaches zero for the $(H / L)^{2} \ll 1$ and $b$ for $(H / L)^{2} \gg 1$. As the squared aspect ratio $(H / L)^{2}$ decreases, the flow becomes more hydrostatic, hence developing less vertical acceleration.

As shown in Table 2, the variations of $H, L$, and $(H / L)^{2}$ between all four simulations (125-m, 250-m, 500-m, and 1000-m cases) are listed. These values are obtained as follows: the $y$-averaged $w$ field and its central maximum $w_{\max }$ are computed to identify the updraft, which is defined as interconnected points with $\bar{w}>0.1 w_{\max }$, at each model output time step. The updraft depth $H$ is then evaluated as the time averaged (05:00 to 06:00) maximum depth of the updraft and the updraft width $L$ is evaluated as the time averaged width of updraft, vertically averaged over the updraft depth.

\begin{tabular}{cccc}
\hline Simulation & $L(\mathrm{~km})$ & $H(\mathrm{~km})$ & $(H / L)^{2}$ \\
\hline$\Delta=125 \mathrm{~m}$ & 1.1 & 1.4 & 1.7 \\
$\Delta=250 \mathrm{~m}$ & 0.9 & 1.6 & 3.4 \\
$\Delta=500 \mathrm{~m}$ & 1.0 & 1.4 & 2.0 \\
$\Delta=1000 \mathrm{~m}$ & 1.9 & 1.3 & 0.5 \\
\hline
\end{tabular}

Table 2: Comparison of subcloud updraft width $(L)$ and depth $(H)$, and squared aspect ratio $\left((H / L)^{2}\right)$ between four simulations with different horizontal grid spacings.

Consistent with the qualitative description of updraft structures in Fig. 10, the 250-m run has the smallest $L$ and largest $H$, hence the largest updraft aspect ratio of the four cases. In particular, the squared updraft aspect ratio $(H / L)^{2}$ in the $250-\mathrm{m}$ run is around twice as large as that in the 125-m and 500-m runs, and around 7 times larger than that 
in the 1000-m run, making it the most nonhydrostatic case among them all. Substituting these $(H / L)^{2}$ into $(20)$, the vertical acceleration in the 250 -m run is about $20 \%$ larger than that in the 125-m and 500-m runs, and well over double than that in the $1000-\mathrm{m}$ run. Although the differences in vertical acceleration between the three highest-resolution runs are not as dramatic as that between the 250-m and 1000-m runs, they still suffice to explain a significant reduction in updraft strength in the $125-\mathrm{m}$ and $500-\mathrm{m}$ runs, relative to the $250-\mathrm{m}$ run.

\subsubsection{The role of turbulence}

The findings presented thus far indicated that the narrower updraft in the 250-m case undergoes stronger upward acceleration than do the wider updrafts in other cases. However, the underlying mechanism responsible for the changes in updraft morphology with respect to $\Delta$ remains unresolved.

While the widening of updraft in low-resolution runs (500-m and 1000-m cases) may be ascribed to an inability to resolve the narrow $(L \sim 1 \mathrm{~km})$ updraft adequately on the model grid, a different mechanism, namely increase in turbulent mixing, is invoked to explain the widening of updraft in the 125-m run.

The vigor of turbulence can be expressed in terms of total turbulent kinetic energy $\left(\mathrm{TKE}_{\text {tot }}\right)$, which can be partitioned in subgrid $\left(\mathrm{TKE}_{\mathrm{sgs}}\right)$ and resolved $\left(\mathrm{TKE}_{\mathrm{res}}\right)$ components. The former is provided by the turbulence parameterization scheme and the latter is computed explicitly as

$$
\mathrm{TKE}_{\mathrm{res}}=\frac{1}{2}\left(u^{\prime 2}+v^{\prime 2}+w^{\prime 2}\right)
$$

where $u^{\prime}, v^{\prime}$, and $w^{\prime}$ are perturbations from the local $y$-average. Vertical profiles of $\mathrm{TKE}_{\text {tot }}$ (averaged over the CCR and over 05:00 to 06:00) are shown in Fig. 15. Evidently, $\mathrm{TKE}_{\text {tot }}$ exhibits strong sensitivity to $\Delta$. In particular, $\mathrm{TKE}_{\text {tot }}$ increases monotonically with decreasing $\Delta$ over the subcloud $(0-1 \mathrm{~km})$ layer, suggesting that stronger turbulent mixing should be anticipated in simulations with smaller horizontal grid spacings. 


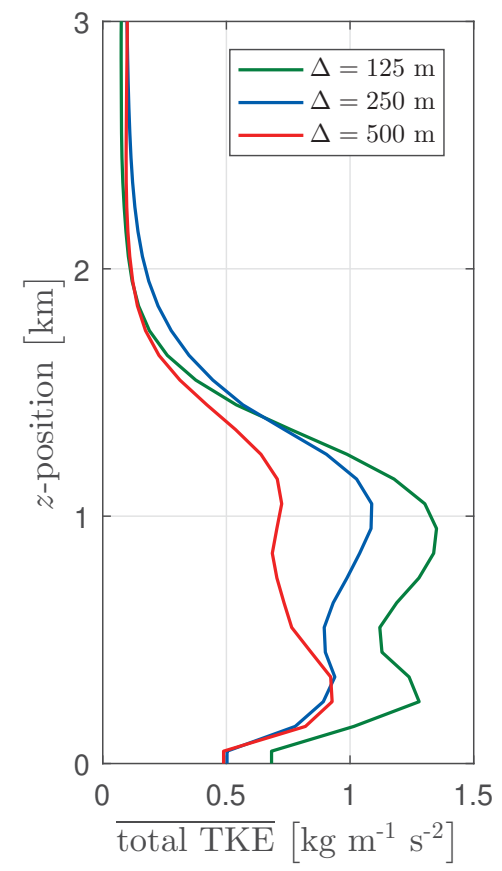

Figure 15: Profiles of total TKE $\left(\mathrm{TKE}_{\mathrm{tot}}=\mathrm{TKE}_{\mathrm{sgs}}+\mathrm{TKE}_{\mathrm{res}}\right)$, averaged temporally from 05:00 to 06:00 and spatially over the CCR, for the three highest-resolution runs.

The impacts of both resolved and subgrid turbulence on the mesoscale circulation can be quantitatively evaluated using the $w$ and zonal wind $(u)$ equations. The former corresponds to the direct effect of turbulence on the updraft and the latter corresponds to the indirect effect of turbulence on low-level convergence, which influences $w$ through the mass-conservation equation.

\subsubsection{Vertical velocity}

The dynamics of subcloud updraft can be represented by a slightly simplified form of (6), where the second term of ADV is eliminated by the anelastic approximation. Applying Reynold's averaging on the resultant equation, in which variables are represented as a sum of a $y$-averaged mean (e.g., $\overline{\mathbf{u}}(x, z, t))$ and perturbation (e.g., $\left.\mathbf{u}^{\prime}(x, y, z, t)\right)$ components. The former represents the mesoscale circulation and the latter represents the turbulent fluctuations. Taking the $y$ - average then gives

$$
\frac{\partial \bar{w}}{\partial t} \approx-\frac{1}{\rho_{0}} \nabla \cdot\left(\rho_{0} \overline{\mathbf{u} w}\right)-c_{p} \overline{\theta_{\rho}^{\prime} \frac{\partial \pi^{\prime}}{\partial z}}+g \frac{{\overline{\theta_{\rho}^{\prime}}}_{\theta_{\rho 0}}}{{ }^{\prime}} \bar{T}_{h, \mathrm{res}}+\bar{T}_{v, \mathrm{res}}+\bar{T}_{h, \mathrm{sgs}}+\bar{T}_{v, \mathrm{sgs}}+\bar{D}_{h}+\bar{D}_{v}
$$


where the horizontal $\left(T_{h, \text { res }}\right)$ and vertical $\left(T_{v, \text { res }}\right)$ parts of resolved turbulence are given by

$$
\begin{aligned}
& \bar{T}_{h, \text { res }}=-\nabla_{h} \cdot\left(\overline{\mathbf{u}^{\prime} w^{\prime}}\right) \\
& \bar{T}_{v, \text { res }}=-\frac{1}{\rho_{0}} \frac{\partial}{\partial z}\left(\rho_{0} \overline{w^{\prime}}\right)
\end{aligned}
$$

Aside from the $y$-averaging, this formulation is similar to (6), in that BUOY and VPPG terms from (6) are unchanged. The advection term in (23) now only represents the advection of mean circulation by the mean flow. The additional resolved turbulence terms derive from the product of perturbation terms in ADV from (6).

For the $125-\mathrm{m}$ and $250-\mathrm{m}$ cases, the magnitude of the turbulent forcing is dominated by the resolved component rather than the subcgrid turbulence (not shown), interpretation of $\bar{T}_{h \text {,res }}$ and $\bar{T}_{v \text {,res }}$ are sufficient to understand the total turbulence $\left(T_{\text {tot }}\right)$ in these cases. Before analyzing the turbulent forcings in (23) in detail, the turbulent fluxes themselves are interpreted. In Fig. 16d, the vertical flux $\overline{w^{\prime 2}}$ in the 250-m run is positive and maximized on the flanks of the updraft at $z \approx 1 \mathrm{~km}$, which corresponds to the height of maximum $\mathrm{TKE}_{\text {tot }}$ shown in Fig. 15. The zonal flux $\overline{u^{\prime} w^{\prime}}$ is more complex, which comprises a dipole centered at $x=0 \mathrm{~km}$ within the core of the central updraft, positive fluxes on the left downdraft, and negative fluxes on the right downdraft (Fig. 16c).

With the emphasis of the current analysis placed on explaining factors governing the magnitude and width of the updraft, physical interpretation of the turbulent fluxes of $w^{\prime}$ is focused on the core within the updraft and its immediate surroundings. Based on the mesoscale flow (shown in blue arrows) in Fig. 10b, negative vertical shear in zonal wind is found on the left of the updraft (i.e. $\partial \overline{\mathbf{u}} / \partial z<0$ ) and positive vertical shear in zonal wind is found on the right of the updraft (i.e. $\partial \overline{\mathbf{u}} / \partial z>0$ ). Hence, the negative/positive flux couplet within the lower updraft is countergradient, while the surrounding fluxes are downgradient.

While the downgradient fluxes within the downdrafts are expected within turbulent layers, the countergradient fluxes in the close vicinity of the updraft likely arise because of small zonal displacements of the central updraft along the convergence line. Considering an updraft that is shifted to the left at a given $y$-location, strongly ascending winds just to the right of $x=0 \mathrm{~km}$ are replaced by more zonal (easterly) flow, hence, $w^{\prime}<0$ and $u^{\prime}<0$ with respect to the $y$-averaged flow over there. Simultaneously, just to the left 
of $x=0 \mathrm{~km}$, the wind perturbations become $w^{\prime}>0$ and $u^{\prime}<0$ there. If the updraft is shifted to the right, the signs of $u^{\prime}$ and $w^{\prime}$ on both sides reverse but the sign of $u^{\prime} w^{\prime}$ remains the same. Thus, the lateral displacement of central updraft induces negative (positive) $y$-averaged $\overline{u^{\prime} w^{\prime}}$ on the left (right) flank of the central updraft.
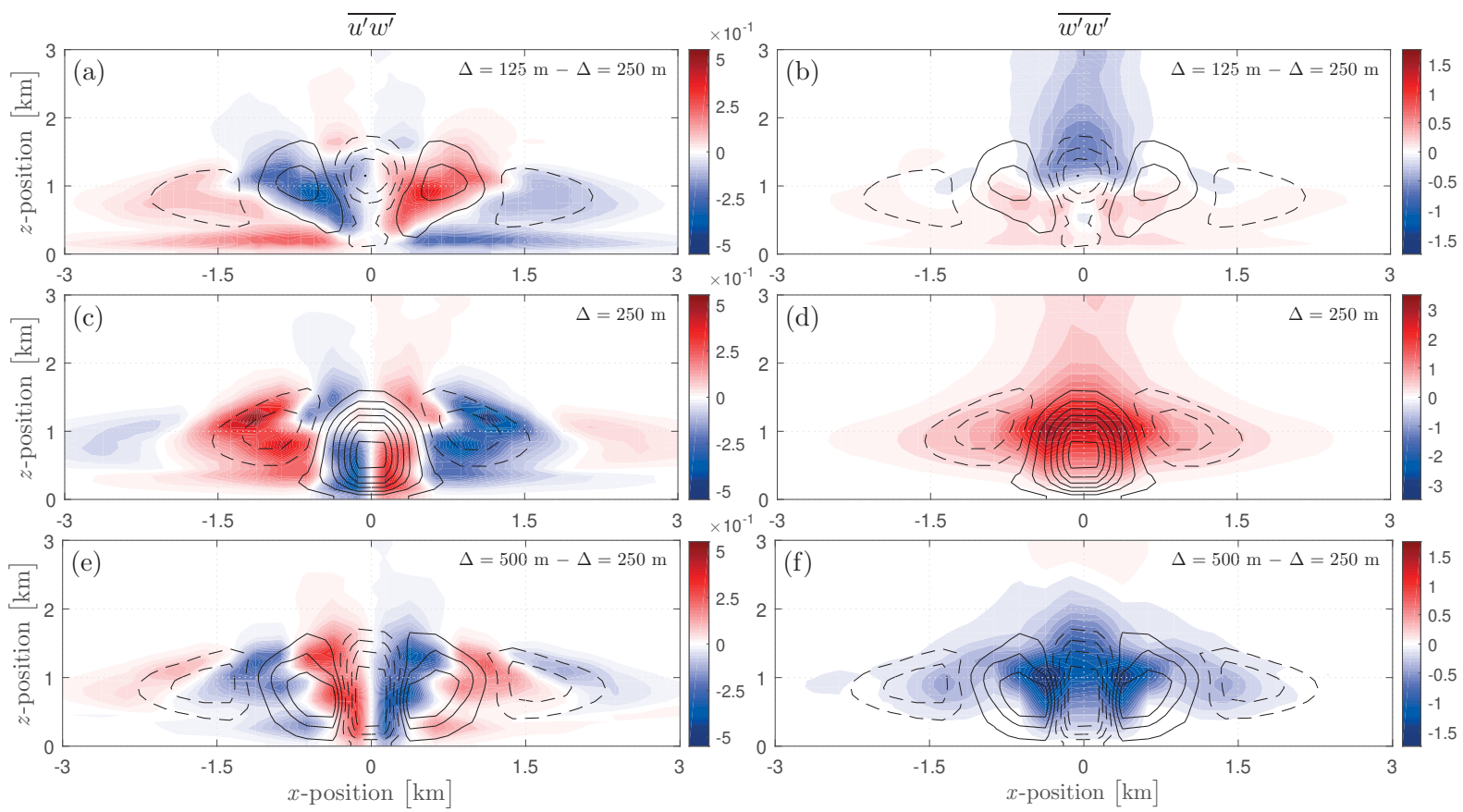

Figure 16: Distributions of $y$-averaged resolved turbulent fluxes of (a), (c), (e) $u^{\prime} w^{\prime}$ and (b), (d), (f) $w^{\prime} w^{\prime}$. The absolute fluxes at $\Delta=250 \mathrm{~m}$ are shown at (c) and (d). The difference of resolved fluxes between $\Delta=125 \mathrm{~m}$ and $\Delta=250 \mathrm{~m}$ are shown in (a-b) and between $\Delta=500$ $m$ and $\Delta=250 m$ are shown in (e-f)

The total turbulent forcings on $w$ in the $250-\mathrm{m}$ case is characterized by a vertical dipole with negative forcing below $z \approx 1 \mathrm{~km}$ and positive forcing above it (Fig. 17b). As the vertical component of this forcing is much larger than the horizontal (Figs. 18c-d), insights into the formation of this dipole can be deduced from the distribution of $\overline{w^{\prime 2}}$ in Fig. 16d, which is maximized at $z=1 \mathrm{~km}$, the height of $\mathrm{TKE}_{\text {tot }}$ maximum. Hence, the vertical gradient of $\overline{w^{\prime 2}}$ is accordingly positive below this height and negative above it. The total turbulent forcings on $w$ is therefore negative below $1 \mathrm{~km}$ and positive above it. At any given height, the sign of this term depends on whether there is a convergence or divergence of turbulent flux of $w^{\prime}$ in the layer. Namely, when this term is negative, it means that there is a net divergence of turbulent flux of $w^{\prime}$ out of the layer (Fig. 17b). Thus, the primary effect of this term on (23) is to transport $w^{\prime}$ near the surface to the upper portion of the updraft, redistributing $w^{\prime}$ perturbations within the subcloud layer.

The horizontal component of the turbulent forcing on $w$ in the $250-\mathrm{m}$ run is negative 
within the updraft core, positive at $x \approx \pm 0.5 \mathrm{~km}$, and then negative at $x \approx \pm 1.5 \mathrm{~km}$ (Fig. 18c). As the contribution of $\overline{v^{\prime} w^{\prime}}$ to this forcing is small(not shown), this pattern can be largely explained by the distribution of $\overline{u^{\prime} w^{\prime}}$, which varies from positive to negative at $x \approx 0.5 \mathrm{~km}$, negative to positive at $x=0 \mathrm{~km}$, and from positive to negative again at $x \approx 0.5 \mathrm{~km}$ (Fig. 16c). As a result, the horizontal component of turbulent forcing on $w$ is negative at the centre of the updraft and positive on the flanks of it (Fig. 18c). The effect of this forcing on (23) is to suppress the $w$ field at $x=0 \mathrm{~km}$ by zonally transporting velocity perturbations to the updraft flanks, which tends to weaken and widen the updraft.

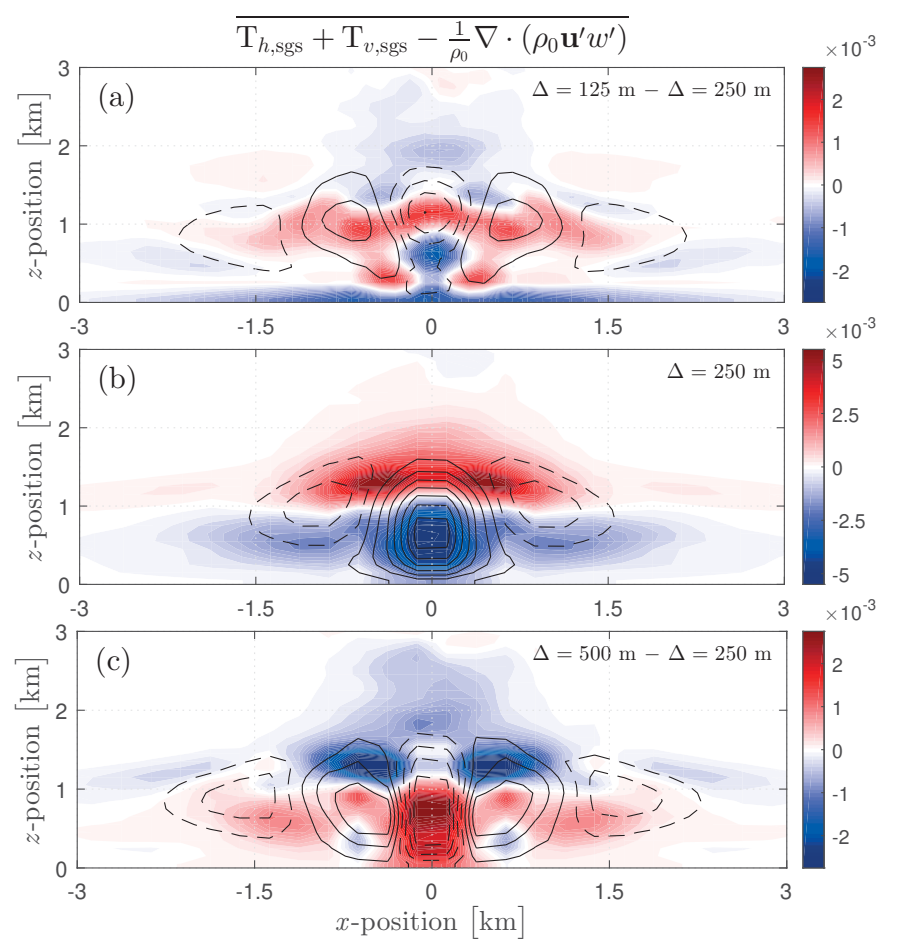

Figure 17: Total (subgrid plus resolved) turbulent forcing on $w$ for (a) $\Delta=125 m-\Delta=$ $250 m$, (b) $\Delta=250 m$, and (c) $\Delta=500 m-\Delta=250 m$

Differences in total turbulent forcing on $w$ between the 125-m and 250-m cases are shown in Fig. 17a, which can also be explained by the variations in turbulent fluxes $\left(\overline{u^{\prime} w^{\prime}}\right.$ and $\left.\overline{w^{\prime 2}}\right)$ themselves between the two cases. In the vertical, the magnitude of $\overline{w^{\prime 2}}$ in the $125-\mathrm{m}$ case is slightly enlarged below $z=1 \mathrm{~km}$ but substantially diminished aloft (Fig. 16b). This suggests that low-level turbulence is intensified and the height of maximum $\overline{w^{\prime 2}}$ is shifted downward, the latter of which is consistent with the decrease in the height of $\mathrm{TKE}_{\text {tot }}$ maximum in the 125-m case shown in Fig. 15. Hence, the resultant vertical component of turbulent forcing on $w$ is more positive and widespread near $z=1$ 

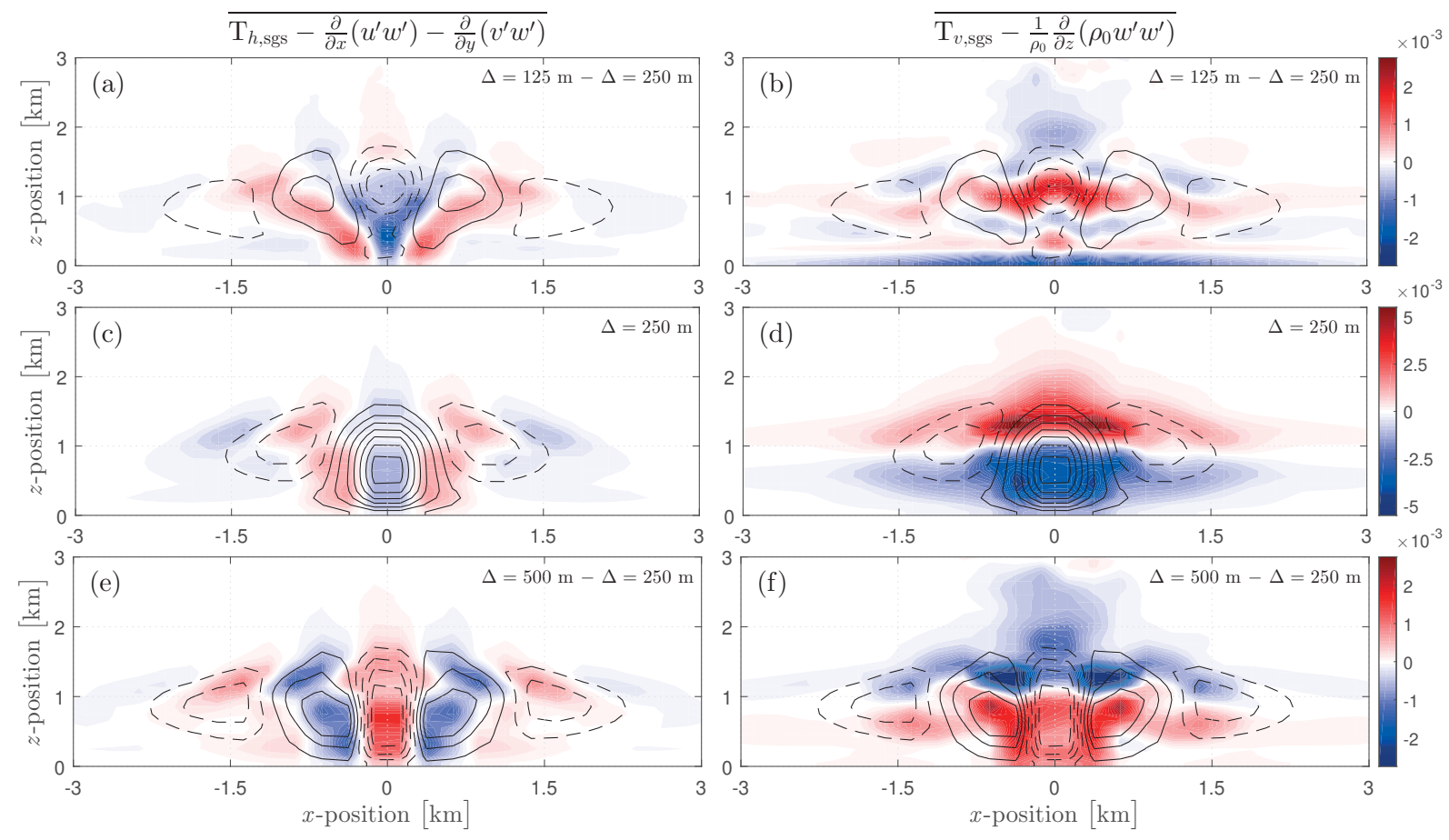

Figure 18: As in Fig. 17, but for the (a), (c), (e) horizontal and (b), (d), (f) vertical components of the total turbulent forcing on $w$

km, and more negative above and below (Fig. 18b), suggesting a shallower updraft with more vigorous turbulent mixing near the surface.

In the horizontal, the 125 -m run exhibits stronger countergradient flux $\left(\overline{u^{\prime} w^{\prime}}\right)$ on the flanks of the updraft (Fig. 16a), which strengthens the negative forcing in the updraft core and the positive forcing on the updraft flanks (Fig. 18a). In particular, the enhancement of this forcing aligns very well with the differences in $y$-averaged vertical velocity $(\bar{w})$ contour lines, suggesting that the horizontal component of turbulent forcing on $w$ reduces vertical velocity within the updraft core but increases $\bar{w}$ on the updraft flanks, due to zonal transport of velocity perturbations. Combining both horizontal and vertical components together generates negative differences in total turbulent forcing on $w$ within the updraft core, with positive differences surrounding it, suggesting a stronger turbulent weakening and widening of the updraft than that in the 250-m case.

In contrast, the differences in the distribution of turbulent fluxes (Fig. 16e-f) and turbulent forcings on $w$ (Fig. 17c and Fig. 18e-f) in the 500-m and 250-m runs are largely similar but of opposite sign to the pattern established in the $250-\mathrm{m}$ case. This can be explained by the reduced turbulence associated with the 500-m case, which has the weakest $\mathrm{TKE}_{\text {tot }}$ among the three (Fig. 15). Thus, the turbulent mixing in the 500-m case is limited and only weakly affects the subcloud mesoscale circulation. 
The effect of turbulence on widening and weakening the central updraft stems from the divergence term embedded in the formulation of turbulent forcing, in which velocity perturbations are transported away from a given location if the term (total turbulent forcing on $w$ ) is negative. Fig. 17b clearly demonstrates that the turbulent forcing acts against the $w$ field in the lower portion of the central updraft. The associated deceleration, particularly along the centerline and within the 0-500 $\mathrm{m}$ layer, can be roughly estimated by applying (8) with an additional turbulent forcing term included, which is

$$
\frac{1}{2} \frac{\partial}{\partial z}\left(\bar{w}^{2}\right) \approx \bar{b}-c_{p} \overline{\theta_{\rho}^{\prime} \frac{\partial \pi^{\prime}}{\partial z}}+\overline{T_{\text {tot }}}
$$

where $T_{\text {tot }}$ represents the combined horizontal and vertical subgrid and resolved turbulent forcing.

Similar to the analysis described by (7)-(8), we roughly estimate the deceleration caused by the negative turbulent forcing in the $250-\mathrm{m}$ case over $0-500 \mathrm{~m}$. To this end, we carry over the LHS from (8) to the present analysis and assume that this value represents the acceleration in the presence of turbulence. Then, we subtract the estimated mean turbulent forcing over $0-500 \mathrm{~m}\left(-5 \times 10^{-3} \mathrm{~ms}^{-2}\right.$; Fig. 17b) from the RHS of (26) and recalculate $\left.w\right|_{500 \mathrm{~m}}$. The result $\left(3.7 \mathrm{~ms}^{-1}\right)$ is substantially larger than the diagnosed value of $\left.w\right|_{500 m}\left(3.0 \mathrm{~ms}^{-1}\right)$. Hence, turbulence significantly modifies the updraft strength and morphology, and contributes meaningfully to the differences in updraft characteristics between the $125-\mathrm{m}$ and $250-\mathrm{m}$ runs.

\subsubsection{Horizontal velocity}

The zonal velocity $\bar{u}$ and vertical velocity $\bar{w}$ in the subcloud layer can be related by the $y$-averaged anelastic mass-continuity equation:

$$
\frac{\partial \bar{u}}{\partial x}+\frac{1}{\rho_{0}} \frac{\partial}{\partial z}\left(\rho_{0} \bar{w}\right)=0
$$

which suggests that changes in $\partial \bar{u} / \partial x$, hence low-level convergence, could lead to changes in $\bar{w}$. The effect can be assessed by the Reynolds-averaged momentum equation for $u$ :

$$
\frac{\partial \bar{u}}{\partial t} \approx-\frac{1}{\rho_{0}} \nabla \cdot\left(\rho_{0} \overline{\mathbf{u} u}\right)-c_{p} \overline{\theta_{\rho}^{\prime} \frac{\partial \pi^{\prime}}{\partial x}}+\bar{T}_{h, \mathrm{res}}+\bar{T}_{v, \mathrm{res}}+\bar{T}_{h, \mathrm{sgs}}+\bar{T}_{v, \mathrm{sgs}}+\bar{D}_{h}+\bar{D}_{v},
$$


where the resolved turbulence terms are defined identically to those in (24)-(25), except for $w^{\prime}$ being replaced by $u^{\prime}$.

The total turbulent forcing on $u$ in the $250-\mathrm{m}$ run is the strongest near the surface, with negative values for $x<0 \mathrm{~km}$ and positive values for $x>0 \mathrm{~km}$ (Fig. 19b). The turbulent forcing not only slows down the converging flow near the domain centre but also opposes the outflow and widens the downdraft near the updraft top.

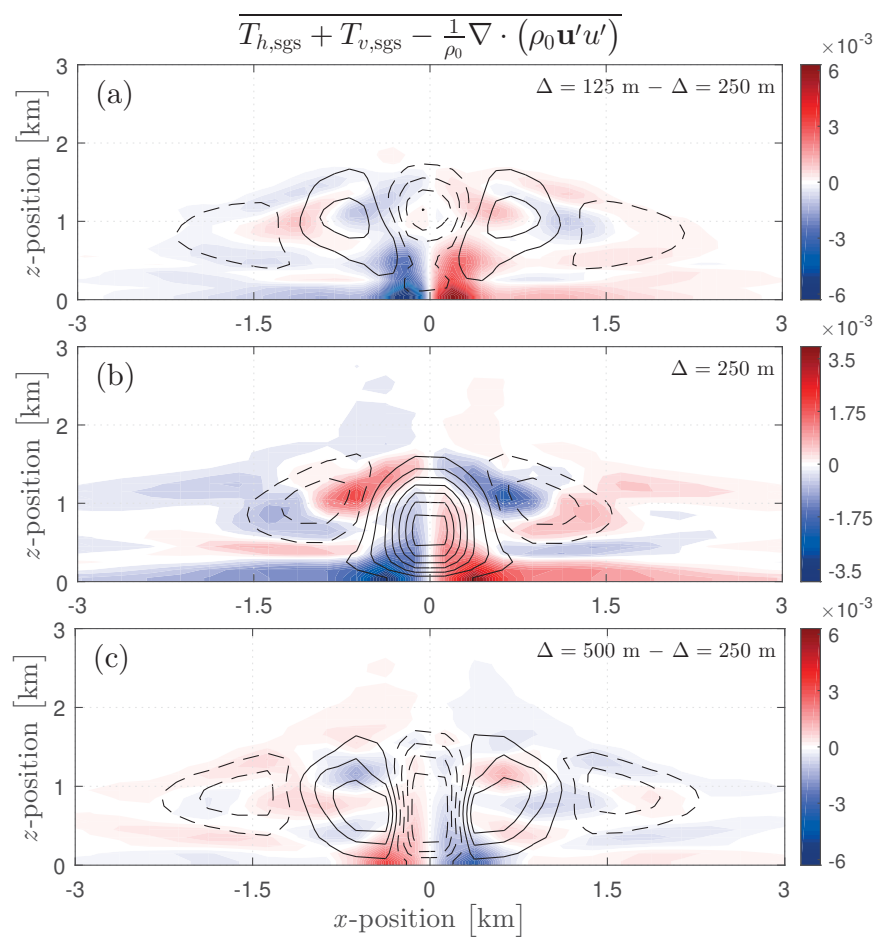

Figure 19: As in Fig. 17, but for turbulent foricng on $u$.

Assuming steady-state mesoscale flow as before, the significance of turbulent forcings on $u$ can be estimated by applying a simplified form of (28) on a parcel that moves horizontally along the surface $(\bar{w}=0)$, with only the terms of zonal perturbation pressure gradient and total turbulence considered:

$$
\frac{1}{2} \frac{\partial}{\partial x}\left(\bar{u}^{2}\right) \approx-c_{p} \overline{\theta_{\rho}^{\prime} \frac{\partial \pi^{\prime}}{\partial x}}+\overline{T_{\mathrm{tot}}}
$$

For constant gradient of $\bar{u}$ over the $x$ interval $[-3,0]$, where the zonal winds $(\mathbf{u} \approx u)$ decreases from $\left.u\right|_{x=-3 k m} \approx-4 \mathrm{~ms}^{-1}$ to $\left.u\right|_{x=0 k m} \approx 0 \mathrm{~ms}^{-1}$, the LHS of (29) is estimated as $2.7 \times 10^{-3} \mathrm{~ms}^{-2}$, which is on the same order of magnitude as the turbulent forcing over the same region (Fig. 19b). Thus, the turbulent forcing term in (28) contributes significantly to the deceleration, and hence convergence, of surface-based inflow. 
Substantial enhancement in turbulent forcing on $u$ is simulated in the 125-m run over the regions near the domain centerline (Fig. 19a). Moreover, the differences in forcing between the $125-\mathrm{m}$ and $250-\mathrm{m}$ runs are much larger in magnitude than that in the $250-\mathrm{m}$ run alone, suggesting that deceleration of mesoscale inflow near the surface, particularly within the updraft flanks, owing to turbulent forcing is considerably larger in the 125-m run. Thus, stronger gradient in turbulent forcing on $u$ along the $x$-axis is developed with the region of low-level convergence shifted laterally outward from the centre, which enhances $\partial w / \partial z$ within the updraft flanks and decreases $\partial w / \partial z$ within the updraft core. Given that $w=0 \mathrm{~ms}^{-1}$ at the surface, the $w$ field changes correspondingly to the changes in surface convergence. Thus, as with those on $w$, the turbulent forcing on $u$ alters the updraft by weakening and widening it.

In the 500-m run, the distribution of turbulent forcing on $u$ is similar to those in the 250-m run but noticeably weaker. Comparable to the changes in turbulent forcing on $w$, these changes on $u$ can also be attributable to the reduced turbulence in this case, which leads to less turbulent deceleration, hence less convergence, on the surface-based inflow prior to ascent. However, the 500-m run contains the strongest numerical diffusion among the three (not shown), which performs a similar function as the turbulence does in the more turbulent, higher-resolution cases.

\section{Conclusions}

Cloud-resolving simulations with the CM1 model were employed to investigate the sensitivity of moist convection initiation to horizontal grid resolution $(\Delta)$. Simulations were initialized with sounding representative of quiescent midlatitude conditionally unstable flow, and forced by a prescribed diurnal surface heating function with peak amplitude located at the domain centerline $(x=0 \mathrm{~km})$. The heating function was symmetric in $x$ and homogeneous in $y$. The former generated locally strong horizontal convergence and a subcloud updraft that breached the level of free convection (LFC) to initiate cumulus convection. For the simulations, horizontal grid spacing was systematically varied from $125 \mathrm{~m}$, to $250 \mathrm{~m}$, to $500 \mathrm{~m}$, and to $1000 \mathrm{~m}$. Most of the analysis was confined to the first three of these simulations, for brevity.

The most distinctive feature from the simulations was that the ultimate height of cloud was significantly higher in the $250-\mathrm{m}$ and $500-\mathrm{m}$ runs $(8-9 \mathrm{~km})$ than in the $125-\mathrm{m}$ run $(6-7$ 
$\mathrm{km}$ ), which also exhibited the smallest vertical mass flux within cloud cores (ascending and positively buoyant cloudy air) at all vertical levels among the three cases. With the largest vertical core mass flux at the LFC found in the 250-m case, the associated subcloud updraft was the most vigorous in that case.

Within the cloud layer, variations in conditionally averaged core properties (vertical velocity, buoyancy, and hydrometeor content) across different horizontal grid spacings were rather limited, except in the vicinity of cloud top. While the conditionally averaged fractional entrainment rates $(\bar{\epsilon})$ were similar between the three cases, midlevel (from the LFC up to $4.5 \mathrm{~km}$ ) fractional detrainment rate $(\bar{\delta})$ was noticeably weaker in the $500-\mathrm{m}$ run than in the other two finer-resolution cases (125-m and 250-m runs). More importantly, $\bar{\delta}$ exceeded $\bar{\epsilon}$ at most vertical levels, leaving the core mass flux to decrease with height. However, the 500-m case did so the least rapidly than the other two cases. Thus, the core mass flux profile of the 500-m run overtook that of the 250-m run slightly aloft cloud base and then remained the strongest afterwards.

Analysis of parcel trajectories revealed that parcels lifted through the subloud layer were increasingly struggling to cross the lifted condensation level (LCL) and reach the middle-to-upper troposphere as $\Delta$ was varied from $250 \mathrm{~m}$. Not only did the $125-\mathrm{m}$ run exhibit the lowest vertical velocity at the LCL $\left(w_{\mathrm{LCL}}\right)$, the smallest fraction of its parcels were able to cross the LFC and became convective cores. Although the mean $w_{\mathrm{LCL}}$ of parcels in the 500-m run was less than that in the $250-\mathrm{m}$ run, those parcels above the LFC in the 500-m run were more likely to attain higher altitudes, which is consistent with the reduced detrainment in the 500-m case.

The cloud-layer and parcel-trajectory analyses both emphasized the importance of $w_{\mathrm{LCL}}$ in determining the core mass flux at the LFC and the ultimate height of cloud. The subcloud vertical velocity budget was therefore studied in detail to identify factors controlling the strength and structure of the subcloud updraft. The buoyancy $(b)$ distribution suggested that the thermal circulations in the $125-\mathrm{m}$ and $500-\mathrm{m}$ runs were clearly weaker and shallower than that in the 250-m run, in that $b$ was larger above the central updraft in the stably stratified layer and smaller along the flanks of the updraft in these two cases. Moreover, the magnitude of adverse vertical perturbation pressure gradient (VPPG) within the lower half of the buoyant updraft was substantially larger in the $125-\mathrm{m}$ and 500-m runs than in the 250-m run, indicating that the flow in the former two 
cases exhibited weaker subcloud forcing for ascent.

Analysis of the updraft morphology showed that subtle differences in updraft length $(L)$ and updraft depth $(H)$ led to notable differences in squared aspect ratios $\left((H / L)^{2}\right)$ between the cases. Namely, the ratios in the $125-\mathrm{m}$ and $500-\mathrm{m}$ runs were half of that in the 250-m run, leading to a substantial reduction in vertical acceleration in the former two cases. This indicates that the updraft in the $250-\mathrm{m}$ case was more nonhydrostatic than that in the other cases.

While the widening and weakening of updraft in low-resolution runs (500-m and 1000m cases) were very likely a result of the narrow $(L \approx 1 \mathrm{~km})$ updraft being inadequately resolved on the model grids, the same reasoning was not applicable to the 125-m run. With considerably larger total (subgrid plus resolved) turbulent kinetic energy (TKE) found at most vertical levels within the subcloud layer in the 125-m run than in the 250$\mathrm{m}$ run, the mechanism behind the weakening and widening of the central updraft as $\Delta$ varied from $250 \mathrm{~m}$ was analyzed from the perspective of turbulent forcing using Reynoldsaveraged momentum equations. In the vertical velocity $(w)$ equation, total turbulent forcing on $w$ directly reduced vertical motion within the updraft core but increased $w$ on the updraft flanks, due to zonal transport of velocity perturbations. In the zonal velocity $(u)$ equation, total turbulent forcing on $u$ decelerated the surface-based inflow near the domain centreline $(x=0 \mathrm{~km})$ and indirectly strengthened $w$ within the updraft flanks through enhanced low-level convergence there. Enhanced turbulent forcings on both $w$ and $u$ were found in the 125-m run, compared to the 250-m run. In particular, the turbulent forcing on $u$ in the $125-\mathrm{m}$ run was more than double that in the $250-\mathrm{m}$ run. This enhanced forcing contributed significantly to a widening and weakening of the central updraft in the 125-m case.

Overall, the intensity of moist convection decreased when horizontal grid spacing was varied from $250 \mathrm{~m}$. Subcloud updrafts were widened and weakened in the 125-m and 500$\mathrm{m}$ cases. Such distortion of updraft structure was ascribed to increase in turbulent fluxes on both $w$ and $u$ fields in the 125-m case, and limitations in resolving the narrow updraft sufficiently on the 500-m model grids. Their subcloud updrafts were more hydrostatic and subject to less vertical acceleration, hence generating much weaker vertical core mass flux near the LFC than the 250-m case. Furthermore, midlevel detrainment was notably weaker in the 500-m run, which led to the slowest decay in core mass flux with height 
among the three. Consequently, the 250-m and 500-m cases developed radically deeper convection than the 125 -m case.

The results presented herein suggest that simulation of deep-convection initiation may be inadequately represented even on a 250-m horizontal grid, as no systematic trends in subcloud forcing and convective mass flux were established when horizontal grid spacing was decreased from $500 \mathrm{~m}$ to $250 \mathrm{~m}$, and to $125 \mathrm{~m}$, leaving uncertainty in whether the 125$\mathrm{m}$ case should be taken as the truth when evaluating the coarser-resolution simulations. The lack of convergence in statistical properties of the thermally induced subcloud updraft arises mainly due to monotonically increasing turbulence as $\Delta$ decreases. This suggests that either the simulated flow was not turbulent enough to include the majority of the energy-containing eddies or the subgrid turbulence model did not respond properly as $\Delta$ varied. While the deficiency of representing convection with horizontal length scales of roughly $1 \mathrm{~km}$ in simulations with comparable grid spacing remains unsettled, the intensity of subcloud-layer turbulent fluxes in these simulations is likely underestimated when compared to their finer-resolution counterparts, if the same subgrid turbulence scheme that was adopted in our study is implemented. Thus, future work could be devoted to identifying the maximum allowable $\Delta$ at which the updraft characteristics are properly resolved, and improving the partitioning of subgrid and resolved components of turbulence in simulated flow. 


\section{References}

Bechtold, P., J.-P. Chaboureau, A. Beljaars, A. Betts, M. Köhler, M. Miller, and J.L. Redelsperger, 2004: The simulation of the diurnal cycle of convective precipitation over land in a global model. Quart. J. Roy. Meteor. Soc., 130, 3119-3137, doi:10.1256/qj.03.103.

Bechtold, P., N. Semane, P. Lopez, J.-P. Chaboureau, A. Beljaars, and N. Bormann, 2014: Representing equilibrium and nonequilibrium convection in large-scale models. J. Atmos. Sci., 71, 734-753, doi:10.1175/JAS-D-13-0163.1.

Bennett, L. J., K. A. Browning, A. M. Blyth, D. J. Parker, and P. A. Clark, 2006: A review of the initiation of precipitating convection in the united kingdom. Quart. J. Roy. Meteor. Soc., 132, 1001-1020, doi:10.1256/qj.05.54.

Bennett, L. J., and Coauthors, 2011: Initiation of convection over the black forest mountains during cops iop15a. Quart. J. Roy. Meteor. Soc., 137, 176-189, doi:10.1002/qj.760.

Betts, A. K., 1975: Parametric interpretation of trade-wind cumulus budget studies. J. Atmos. Sci., 32, 1934-1945, doi:10.1175/15200469(1975)032<1934:PIOTWC>2.0.CO;2.

Bryan, G. H., and J. M. Fritsch, 2004: A reevaluation of ice-liquid water potential temperature. Mon. Wea. Rev., 132, 2421-2431, doi:10.1175/15200493(2004)132<2421:AROIWP > 2.0.CO;2.

Bryan, G. H., J. C. Wyngaard, and J. M. Fritsch, 2003: Resolution requirements for the simulation of deep moist convection. Mon. Wea. Rev., 131, 2394-2416, doi:10.1175/1520-0493(2003)131<2394:RRFTSO>2.0.CO;2.

Crook, N. A., 1996: Sensitivity of moist convection forced by boundary layer processes to low-level thermodynamic fields. Mon. Wea. Rev., 124, 1767-1785, doi:10.1175/15200493(1996) $124<1767: \mathrm{SOMCFB}>2.0$.CO;2.

Dawe, J., and P. Austin, 2012: Statistical analysis of an les shallow cumulus cloud ensemble using a cloud tracking algorithm. Atmos. Chem. Phys., 12, 1101-1119, doi:10.5194/acp-12-1101-2012.

Fabry, F., 2006: The spatial variability of moisture in the boundary layer and its effect on convection initiation: Project-long characterization. Mon. Wea. Rev., 134, 79-91, doi:10.1175/MWR3055.1.

Hannah, W. M., 2017: Entrainment versus dilution in tropical deep convection. J. Atmos. Sci., 74, 3725-3747, doi:10.1175/JAS-D-16-0169.1.

Houston, A. L., and D. Niyogi, 2007: The sensitivity of convective initiation to the lapse rate of the active cloud-bearing layer. Mon. Wea. Rev., 135, 3013-3032, doi:10.1175/MWR3449.1.

Kang, S.-L., and G. H. Bryan, 2011: A large-eddy simulation study of moist convection initiation over heterogeneous surface fluxes. Mon. Wea. Rev., 139, 2901-2917, doi:10.1175/MWR-D-10-05037.1.

Khairoutdinov, M., and D. Randall, 2006: High-resolution simulation of shallow-to-deep convection transition over land. J. Atmos. Sci., 63, 3421-3436, doi:10.1175/JAS3810.1.

Kirshbaum, D. J., 2011: Cloud-resolving simulations of deep convection over a heated mountain. J. Atmos. Sci., 68, 361-378, doi:10.1175/2010JAS3642.1.

Kirshbaum, D. J., and A. L. M. Grant, 2012: Invigoration of cumulus cloud fields by mesoscale ascent. Quart. J. Roy. Meteor. Soc., 138, 2136-2150, doi:10.1002/qj.1954. 
Klemp, J. B., and R. B. Wilhelmson, 1978: The simulation of three-dimensional convective storm dynamics. J. Atmos. Sci., 35, 1070-1096, doi:10.1175/15200469(1978)035<1070:TSOTDC > 2.0.CO;2.

Klüpfel, V., N. Kalthoff, L. Gantner, and C. M. Taylor, 2012: Convergence zones and their impact on the initiation of a mesoscale convective system in west africa. Quart. J. Roy. Meteor. Soc., 138, 950-963, doi:10.1002/qj.979.

Kuang, Z., and C. S. Bretherton, 2006: A mass-flux scheme view of a high-resolution simulation of a transition from shallow to deep cumulus convection. J. Atmos. Sci., 63, 1895-1909, doi:10.1175/JAS3723.1.

Lock, N. A., and A. L. Houston, 2014: Empirical examination of the factors regulating thunderstorm initiation. Mon. Wea. Rev., 142, 240-258, doi:10.1175/MWR-D-1300082.1 .

Markowski, P., and Y. Richardson, 2011: Mesoscale meteorology in midlatitudes. Wiley, $430 \mathrm{pp}$.

Morrison, H., J. A. Curry, and V. I. Khvorostyanov, 2005: A new double-moment microphysics parameterization for application in cloud and climate models. part i: Description. J. Atmos. Sci., 62, 1665-1677, doi:10.1175/JAS3446.1.

Rieck, M., C. Hohenegger, and C. C. van Heerwaarden, 2014: The influence of land surface heterogeneities on cloud size development. Mon. Wea. Rev., 142, 3830-3846, doi:10.1175/MWR-D-13-00354.1.

Romps, D. M., and Z. Kuang, 2010: Nature versus nurture in shallow convection. J. Atmos. Sci., 67, 1655-1666, doi:10.1175/2009JAS3307.1.

Rousseau-Rizzi, R., D. J. Kirshbaum, and M. K. Yau, 2017: Initiation of deep convection over an idealized mesoscale convergence line. J. Atmos. Sci., 74, 835-853, doi:10.1175/JAS-D-16-0221.1.

Stratton, R., and A. Stirling, 2012: Improving the diurnal cycle of convection in gcms. Quart. J. Roy. Meteor. Soc., 138, 1121-1134, doi:10.1002/qj.991.

Tompkins, A. M., 2001: Organization of tropical convection in low vertical wind shears: The role of cold pools. J. Atmos. Sci., 58, 1650-1672, doi:10.1175/15200469(2001)058<1650:OOTCIL > 2.0.CO;2.

Varble, A., and Coauthors, 2014: Evaluation of cloud-resolving and limited area model intercomparison simulations using twp-ice observations: 1. deep convective updraft properties. J. Geophys. Res. Atmos., 119, 13-891, doi:10.1002/2013JD021372.

Waite, M. L., and B. Khouider, 2010: The deepening of tropical convection by congestus preconditioning. J. Atmos. Sci., 67, 2601-2615, doi:10.1175/2010JAS3357.1.

Weckwerth, T. M., and D. B. Parsons, 2006: A review of convection initiation and motivation for ihop_2002. Mon. Wea. Rev., 134, 5-22, doi:10.1175/MWR3067.1.

Weisman, M. L., W. C. Skamarock, and J. B. Klemp, 1997: The resolution dependence of explicitly modeled convective systems. Mon. Wea. Rev., 125, 527-548, doi:10.1175/1520-0493(1997)125<0527:TRDOEM>2.0.CO;2.

Wilson, J. W., and W. E. Schreiber, 1986: Initiation of convective storms at radar-observed boundary-layer convergence lines. Mon. Wea. Rev., 114, 2516-2536, doi:10.1175/1520-0493(1986)114<2516:IOCSAR>2.0.CO;2. 